\title{
Licitaciones no balanceadas: Una aplicación a los contratos de construcción del departamento de transporte de Michigan*
}

\author{
María Florencia Ruiz Diaz ${ }^{\dagger}$ \\ Tesis de Maestría \\ Maestría en Economía \\ Universidad Nacional de La Plata \\ Directora de Tesis: Irene Brambilla
}

\begin{abstract}
Resumen
Al momento de presentar los pliegos en una licitación, los contratistas saben que pueden existir renegociaciones en los contratos. Si anticipan que ciertas cantidades estimadas por el ingeniero han sido sub-estimadas (o sobre-estimadas), pueden tener incentivos a desbalancear estratégicamente los precios ofertados con el fin de aumentar los beneficios del proyecto. Se desarrolla un modelo que incorpora este comportamiento estratégico suponiendo que los contratistas son neutrales al riesgo y se lo contrasta usando los contratos de construcción del departamento de transporte de Michigan para el período 2001-2013. Se encuentra evidencia empírica de que los contratistas desbalancean estratégicamente sus precios y obtienen ingresos $1.6 \%$ adicionales a nivel de proyecto. Además, los contratistas con mayor participación en el mercado de licitaciones son quienes lo realizan de manera más agresiva. Por último, se desarrolla de manera teórica un segundo modelo que considera que los contratistas son aversos al riesgo y tienen heterogeneidad en los costos.
\end{abstract}

Clasificación JEL: D82, L74

*Agradezco a mi directora Irene Brambilla y a Paulo Somaini por el apoyo y sus valiosos comentarios. También agradezco a la lectora del trabajo María Florencia Gabrielli por sus comentarios. Los errores u omisiones son total responsabilidad de la autora.

${ }^{\dagger}$ Universidad Nacional de La Plata. Email: ruizdiazflor@gmail.com 


\section{Índice}

1. Introducción $\quad 3$

2. Licitaciones de contratos de construcción $\quad 6$

2.1. Incentivos de los contratistas a desbalancear el pliego . . . . . . . . . . . 7

3. Base de Datos y Estadísticas Descriptivas 9

4. Modelo - Contratistas neutrales al riesgo 11

4.1. Cálculo de las rentas de desbalanceo . . . . . . . . . . . . . . . . . 13

4.2. Evidencia de Front-End loading . . . . . . . . . . . . . . . . . . . 15

4.3. ¿Los contratistas tienen información más precisa que el ingeniero? . . . . . . . . 16

4.4. ¿Los contratistas tienen información más precisa que el consultor? . . . . . . . . 18

4.5. ¿Todos los contratistas ganadores tienen la misma información? . . . . . . . . . . 20

5. Modelo - Contratistas aversos al riesgo $\quad 22$

5.1. Problema del Contratista . . . . . . . . . . . . . . . . . . 23

5.2. Problema del Econometrista . . . . . . . . . . . . . . . . 25

5.3. Identificación del Modelo . . . . . . . . . . . . . . . . 25

6. Conclusión $\quad 27$

$\begin{array}{lr}\text { 7. Bibliografía } & 28\end{array}$

$\begin{array}{ll}\text { A. Anexo } & 30\end{array}$

A.1. Tablas Adicionales . . . . . . . . . . . . . . . . . . . 30

A.2. Metodología de Estimación . . . . . . . . . . . . . . . 33 


\section{Introducción}

Las agencias públicas necesitan adquirir bienes y servicios del sector privado. Una forma de lograrlo es a través de las licitaciones públicas que buscan hacerlo de una manera transparente y competitiva. Cuando se licita un proyecto complejo, es importante limitar el riesgo que enfrentan los contratistas a eventuales cambios en los plazos, dimensión o errores de estimación del proyecto. Por tal razón, muchas agencias utilizan First-price auctions que prevén algún tipo de ajuste o renegociación en el contrato durante la ejecución del proyecto. Existen dos tipos de problemas que preocupan a estas agencias: i) Colusión: cuando dos o más contratistas conspiran para mantener precios altos y ii) Desbalanceo: cuando los contratistas se aprovechan de las reglas de renegociación. En este trabajo se analiza empíricamente la existencia de contratos no balanceados en las licitaciones del Departamento de Transporte de Michigan (MDOT).

Los contratos en el mercado de contrataciones públicas son complejos. Pueden existir diferencias significativas entre las especificaciones del contrato original y el trabajo y materiales que se terminan necesitando al completar el proyecto. Es por ello que al momento de realizarse la licitación, las agencias públicas utilizan First-price auctions que prevén algún tipo de ajuste o renegociación en el futuro. Estos contratos están formados por varios componentes que se van desarrollando en un lapso de tiempo determinado y cuyas cantidades originalmente estimadas por las agencias se pueden renegociar. Por lo tanto, al momento de la licitación los contratistas tienen que decidir: i) el monto total del pliego a ofertar, y ii) cómo distribuir el monto total entre los componentes mediante la asignación de un precio a cada componente del proyecto.

Tradicionalmente, la literatura empírica se ocupó de estudiar los temas relacionados a la elección óptima del monto total del pliego ofertado por los contratistas (First-price auction). En particular, se desarrolló una amplia literatura que utiliza métodos estructurales para recuperar los costos que racionalizan el monto total ofertado ${ }^{1}$. Ahora bien, cuando los contratos se pueden renegociar es interesante también analizar cómo se comportan los contratistas al asignar los precios entre los componentes del proyecto. Para este caso, los contratistas pueden comportarse estratégicamente desbalanceando sus pliegos (en inglés, bid skewing) con el fin de obtener rentas. El trabajo de Athey y Levin (2001) muestra que las rentas pueden estar asociadas con información acerca de posibles errores de estimación de las cantidades de los componentes del proyecto por parte del subastador. Además, los contratistas pueden obtener ventajas financieras si los pagos son realizados conforme a medida que se completa el trabajo (Cattell -1984, 1987 y 2008). Este trabajo analiza la existencia de estas rentas mencionadas por Athey, Levin y Cattell en los contratos de construcción del MDOT.

Los ingenieros del MDOT preparan una lista de ítems (o componentes) que describen las tareas y materiales necesarios para el trabajo. Para cada ítem, los ingenieros proveen una estimación de las cantidades necesarias para completar el trabajo y también proveen un precio por unidad que

\footnotetext{
${ }^{1}$ Se destacan los trabajos de Aryal y Gabrielli (2013), Athey y Levin (2011), Bajari y Ye (2003), Flambard y Perrigne (2006), Guerre et al. (2000 y 2009), Haile, Hong y Shum (2004), Kawai (2011), Krasnokutskaya (2011), Krasnokutskaya y Seim (2008), Laffont y Vuong (1996), Paarsch y Hong (2006), Perrigne y Vuong (1999 y 2008$)$ y Porter y Zona (1999 y 1993) entre otros.
} 
sirve como referencia. Esta lista se anuncia públicamente junto con los planes y especificaciones que describen cómo el proyecto debe completarse. El contratista interesado en el proyecto ofrecerá un precio por unidad para cada ítem de trabajo de la lista del ingeniero. Los pliegos son cerrados y se presentan antes de una fecha determinada. Cuando los pliegos se abren, el contrato se ofrece al contratista con el menor costo estimado del proyecto, definido este como el producto interno de los precios ofrecidos y las cantidades estimadas por el ingeniero. El departamento de transporte pagará a los contratistas a medida que vayan completando las tareas/ítems. Los montos son calculados multiplicando los precios por ítem por las cantidades efectivamente completadas ${ }^{2}$.

El pago final al contratista dependerá de los precios ofrecidos y de las cantidades efectivamente realizadas. Es decir, si en la ejecución del proyecto se requieren más unidades que las presupuestadas por el ingeniero del proyecto, estas unidades adicionales también se pagarán al contratista al precio ofrecido, y en consecuencia el monto total pagado será mayor al del pliego. El contratista puede incrementar estratégicamente los precios ofrecidos en las tareas cuyas cantidades hayan sido subestimadas por el ingeniero. Esta práctica denominada quantity error exploitation o individual rate loading tiene como objetivo incrementar el pago final esperado. El contratista también puede ofrecer precios más elevados en las tareas que deban realizarse en las fases iniciales del proyecto. Esta práctica denominada front-end loading tiene como objetivo obtener tempranamente una mayor proporción del monto total contratado necesario para financiar sus actividades.

La literatura en Construction Management identificó los posibles beneficios de desbalancear los precios ofrecidos en los trabajos de Marvin Gates (1959 y 1967), Stark (1968, 1972 y 1974), Diekmann y otros (1982), Cattell (1984 y 1987) y Cattell y otros (2008 y 2010). El concepto de los contratos de licitaciones no balanceadas fue primeramente comentado por Marvin Gates (1959 y 1967) donde propuso un método que aborda la necesidad del constructor de obtener un flujo de fondos más rápido, como así también los beneficios de incrementar los precios de los ítems cuyas cantidades se espera que se van a incrementar. Stark (1968, 1972 y 1974) modeló los beneficios del front-end loading y del quantity error exploitation. También reconoció el riesgo vinculado a estas dos estrategias. Diekmann y otros (1982) usaron el modelo determinístico de Stark y agregaron una formulación probabilística para incorporar el riesgo. El único riesgo que fue considerado fue el de que las cantidades finales pueden ser diferentes a las que el constructor inicialmente había estimado. Cattell y otros (2010) proponen la aplicación de la teoría moderna del portafolio a los modelos de desbalanceo. Más allá de que hubo un esfuerzo considerable en tratar de modelar las prácticas de front-end loading y de quantity error exploitation en la construcción poco se sabe sobre la aplicación empírica de estos modelos anteriormente descriptos. De los trabajos empíricos que hacen referencia en cierta medida a estas prácticas en la construcción se destaca el de Bajari, Houghton y Tadelis (2011). Ellos muestran que la renegociación en los contratos de pavimentación de las autopistas impone costos de adaptación significativos. Estos generan más distorsiones y fricciones que los mark-ups explicados por el poder de mercado y licitaciones no balanceadas (sólo tienen en cuenta el quantity error exploitation). Existen trabajos que hacen un análisis empírico de los contratos no

\footnotetext{
${ }^{2}$ En este caso, se está en presencia de una Quasi First- price auction: El MDOT elige el pliego más económico y el pago al contratista dependerá de los precios ofrecidos y de las cantidades efectivamente realizadas.
} 
balanceados en otros mercados, como por ejemplo, en el US Forest Service Timber auctions. Athey y Levin (2001) analizan si existe bid skewing debido al quantity error exploitation.

Este trabajo tiene tres objetivos empíricos: i) analizar la existencia de front-end loading en los contratos de construcción del MDOT, ii) documentar la existencia de quantity error exploitation la cual reflejaría asimetrías de la información entre los contratistas y el licitador; y iii) analizar si los resultados de la licitación reflejan asimetrías de la información entre los contratistas.

A pesar de que no se observa la información que tienen los contratistas al momento de realizar sus ofertas, se puede inferir la calidad de la misma comparando los ingresos que obtienen en los datos con los ingresos que obtendrían si ofertaran precios unitarios distintos. Es decir, se compara los retornos que los contratistas efectivamente obtienen con los retornos que obtendrían utilizando las acciones óptimas bajo distintos supuestos informativos acerca de los ajustes de las cantidades. Entonces, si los contratistas obtienen mayores retornos, se concluye que los mismos tienen información más precisa que la supuesta y actúan sobre ella desbalanceando sus pliegos (o haciendo bid skewing). Y si obtienen retornos iguales o inferiores, se concluye que los contratistas no tienen información más precisa que la supuesta.

Se desarrolla un modelo que permite distinguir y contrastar empíricamente las estrategias de front-end loading y quantity error exploitation por separado y supone que los contratistas son neutrales al riesgo. Para la estrategia de quantity error exploitation, el modelo considera que los contratistas obtienen su vector de precios óptimos dado unas creencias o información acerca de los ajustes de cantidades. Se contrastan empíricamente 3 hipótesis acerca de la información del contratista. La primera hipótesis considera que los contratistas no tienen información más precisa que la que posee el ingeniero. Es decir, se comparan los retornos que los contratistas efectivamente obtienen con los retornos que obtendrían si no tuvieran ningún tipo de información acerca de la variación futura de las cantidades. La segunda hipótesis considera que los contratistas no tienen información más precisa que la que posee el consultor. Aquí se comparan los retornos que los contratistas obtienen en los datos con los retornos que obtendrían si conocieran la distribución empírica de los ajustes de cantidades. Y por último, la tercera hipótesis considera que todas las firmas tienen la misma información.

No se encontró evidencia estadísticamente significativa de que los contratistas estén realizando front-end loading, pero sí se encontró evidencia positiva de quantity error exploitation: i) los contratistas obtienen retornos significativamente mayores de los que obtendrían si simplemente re-escalan los precios del ingeniero, ii) los contratistas no obtienen retornos significativamente mayores de los que obtendrían utilizando la distribución empírica, excepto dos de las firmas con mayor cantidad de contratos ganados, y iii) las firmas tienen distinta información (existen dos firmas que desbalancean sus precios de manera más agresiva).

Para finalizar, se presenta una variante al modelo que permite que los contratistas sean aversos al riesgo. Ahora, la heterogeneidad de los costos entre los contratistas y el grado de aversión al riesgo pueden juegar un rol importante y limitar el bid skewing. La estimación de este modelo y su comparación con el modelo anterior están fuera del alcance de esta tesis.

El trabajo se estructura de la siguiente manera. La sección 2 presenta cómo funciona el sistema 
de licitaciones en los contratos de construcción. La sección 3 describe la base de datos y las estadísticas descriptivas. La sección 4 presenta el modelo con los contraste de hipótesis y sus respectivos resultados. La sección 5 presenta el modelo teórico cuando los contratistas son aversos al riesgo. La sección 6 concluye.

\section{Licitaciones de contratos de construcción}

En esta sección se describe el funcionamiento del sistema de licitaciones en los contratos de construcción y se desarrolla un ejemplo que explica intuitivamente las estrategias de quantity error exploitation y front-end loading.

Los ingenieros del MDOT preparan una lista de ítems (o componentes) que describen las tareas y materiales necesarios para el trabajo. En los contratos que se van a analizar algunos de estos ítems incluyen, por ejemplo, la limpieza inicial, la excavación de tierra, la excavación de roca y la limpieza final. Para cada ítem, los ingenieros proveen una estimación de las cantidades necesarias para completar el trabajo y también proveen un precio por unidad que sirve como referencia. Como puede verse en el ejemplo de la Tabla 1, se estima que se necesitan 50.000 yardas cúbicas para la excavación de tierra a un precio 1,6 dólares por unidad. Esta lista se anuncia públicamente junto con los planes y especificaciones que describen cómo el proyecto debe completarse.

Tabla 1: Costo Estimado del proyecto por el Ingeniero

\begin{tabular}{clllrr}
\hline Ítem No & Descripción & Unidad & $\begin{array}{l}\text { Cantidad } \\
\text { Nominal }\end{array}$ & $\begin{array}{l}\text { Precio x unidad } \\
\text { (dólares) }\end{array}$ & $\begin{array}{l}\text { Monto } \\
\text { (dólares) }\end{array}$ \\
\hline 1 & Limpieza inicial & suma fija & 1 & 54.000 & 54.000 \\
2 & Escavar Tierra & yarda cúbica & 50.000 & 1,6 & 81.000 \\
3 & Escavar Roca & yarda cúbica & 25.000 & 3,2 & 81.000 \\
4 & Limpieza final & suma fija & 1 & 54.000 & 54.000 \\
\hline & & & Total & & 270.000 \\
\hline
\end{tabular}

Fuente: Adaptado en base a un ejemplo de Gates (1967).

Los contratistas que deseen realizar una oferta tienen que decidir: i) el monto total del pliego (Tender Price) y ii) la asignación de precios entre los componentes del proyecto tal que el monto total sea igual al producto interno entre los precios ofrecidos y las cantidades estimadas por el ingeniero. Los pliegos son cerrados y se presentan antes de una fecha determinada. Cuando los pliegos se abren, el licitador elige como ganador al contratista que haya ofrecido un monto total más bajo sin prestar atención a la distribución de precios. En el ejemplo de la Tabla 2 el contratista 1 gana la licitación porque ofrece un monto total más bajo que el contratista 2. La distribución de los precios es relevante al momento de pagar el trabajo completado. El departamento de transporte pagará al contratista ganador a medida que vaya completando las tareas/ítems. La cantidad pagada surge de multiplicar los precios ofrecidos por las cantidades efectivamente completadas. Si las cantidades realizadas son iguales a las nominales (estimadas), el monto pagado será igual al monto total. 
En cambio, si hay modificaciones en la cantidades realizadas, el monto pagado dependerá de la asignación de precios entre los componentes. En consecuencia, los contratistas tendrán incentivos a elegir la distribución de precios de manera estratégica.

Tabla 2: Pliegos de dos contratistas

\begin{tabular}{clllrrrr}
\hline \multirow{2}{*}{ Ítem No } & \multirow{2}{*}{ Descripción } & Unidad & Cantidad & \multicolumn{2}{c}{ Contratista 1 } & \multicolumn{2}{c}{ Contratista 2 } \\
\cline { 5 - 8 } & & Nominal & $\begin{array}{l}\text { Precio x unidad } \\
\text { (dólares) }\end{array}$ & $\begin{array}{l}\text { Monto } \\
\text { (dólares) }\end{array}$ & $\begin{array}{l}\text { Precio x unidad } \\
\text { (dólares) }\end{array}$ & $\begin{array}{l}\text { Monto } \\
\text { (dólares) }\end{array}$ \\
\hline 1 & Limpieza inicial & suma fija & 1 & 50.000 & 50.000 & 60.000 & 60.000 \\
2 & Escavar Tierra & yarda cúbica & 50.000 & 1,5 & 75.000 & 2,2 & 110.000 \\
3 & Escavar Roca & yarda cúbica & 25.000 & 3 & 75.000 & 3 & 75.000 \\
4 & Limpieza final & suma fija & 1 & 50.000 & 50.000 & 55.000 & 55.000 \\
\hline & & & \multicolumn{2}{c}{ Total } & 250.000 & 300.000 \\
\hline
\end{tabular}

Fuente: Adaptado en base a un ejemplo de Gates (1967).

\subsection{Incentivos de los contratistas a desbalancear el pliego}

Si existiesen asimetrías de la información entre los contratistas y el licitador en cuanto a los ajustes de cantidades futuros, los contratistas pueden desbalancear (o skew) sus pliegos estratégicamente aplicando diferentes mark-ups a los componentes del proyecto. En primer lugar, pueden obtener tempranamente una mayor proporción del monto total contratado si eligen precios o markups más elevados en las tareas que deban realizarse en las fases iniciales del proyecto. Esta práctica de desbalanceo se denomina front-end loading. En segundo lugar, pueden incrementar el pago final esperado si incrementan los mark-ups a las tareas cuyas cantidades hayan sido subestimadas por el ingeniero del MDOT. Esta práctica se denomina quantity error exploitation o individual rate loading.

Las tablas a continuación presentan dos ejemplos de estas prácticas de desbalanceo. Si los contratistas reciben sus pagos al completar cada uno de los componentes del proyecto, la estrategia de front-end loading les permite obtener una ventaja financiera. Este ejemplo se ilustra en la Tabla 3 donde existen dos pliegos A y B con el mismo monto total ofertado. Sin embargo, el pliego B se encuentra no balanceado ya que el contratista asigna un precio más alto a la limpieza inicial que se encuentra al principio del proyecto y reduce el precio de la limpieza final de manera tal que el monto total a ofertar permanece constante. De este modo, el contratista logra obtener 40,000 dólares adicionales al principio del proyecto los cuales ayudan a financiar sus operaciones. 
Tabla 3: Pliego no balanceado - Front-End Loading

\begin{tabular}{|c|c|c|c|c|c|c|c|}
\hline \multirow[b]{2}{*}{ Ítem No } & \multirow[b]{2}{*}{ Descripción } & \multirow[b]{2}{*}{ Unidad } & \multirow[b]{2}{*}{$\begin{array}{l}\text { Cantidad } \\
\text { Nominal }\end{array}$} & \multicolumn{2}{|c|}{ Pliego A } & \multicolumn{2}{|c|}{$\begin{array}{l}\text { Pliego B } \\
\text { Front End Loading }\end{array}$} \\
\hline & & & & $\begin{array}{l}\text { Precio x unidad } \\
\text { (dólares) }\end{array}$ & $\begin{array}{l}\text { Monto } \\
\text { (dólares) }\end{array}$ & $\begin{array}{l}\text { Precio x unidad } \\
\text { (dólares) }\end{array}$ & $\begin{array}{l}\text { Monto } \\
\text { (dólares) }\end{array}$ \\
\hline 1 & Limpieza inicial & suma fija & 1 & 50.000 & 50.000 & 90.000 & 90.000 \\
\hline 2 & Escavar Tierra & yarda cúbica & 50.000 & 1,5 & 75.000 & 1,5 & 75.000 \\
\hline 3 & Escavar Roca & yarda cúbica & 25.000 & 3 & 75.000 & 3 & 75.000 \\
\hline \multirow[t]{2}{*}{4} & Limpieza final & suma fija & 1 & 50.000 & 50.000 & 10.000 & 10.000 \\
\hline & & & & Total & 250.000 & & 250.000 \\
\hline
\end{tabular}

Fuente: Adaptado en base a un ejemplo de Gates (1967).

En las Tablas 4 y 5 se ilustra con un ejemplo la estrategia de Quantity Error Exploitation. Existe un contratista cuyo costo de excavar tierra es de $\$ 1.2$ por yarda cúbica mientras que el costo de excavar roca es de $\$ 2.4$ por yarda cúbica. La Tabla 4 muestra dos pliegos A y B con el mismo monto total ofertado pero la distribución de mark-ups entre los componentes es diferente. El contratista luego de haber realizado una investigación en el área a llevarse a cabo el proyecto, tiene razones para creer que se realizarán 30,000 yardas cúbicas de excavación de tierra en vez de 50,000, y se realizarán 45,000 yardas cúbicas de excavación de rocas en vez de 25,000. El contratista puede desbalancear el pliego asignando un precio más elevado a la excavación de rocas y un precio más bajo a la excavación de tierra. En este ejemplo, la distribución de mark-ups del Pliego B refleja esta estrategia. En la estrategia de quantity error exploitation, si los contratistas pueden predecir alguna variación en las cantidades estimadas por el ingeniero, ellos pueden fijar precios más altos (bajos) para aquellos componentes que creen que van a aumentar (disminuir).

Tabla 4: Pliego no balanceado - Quantity Error Exploitation

\begin{tabular}{clllrrrr}
\hline & & & \multicolumn{2}{c}{ Pliego A } & \multicolumn{2}{c}{$\begin{array}{c}\text { Pliego B } \\
\text { Quantity Error Exploitation }\end{array}$} \\
\cline { 5 - 8 } Ítem No & Descripción & Unidad & $\begin{array}{l}\text { Cantidad } \\
\text { Nominal }\end{array}$ & $\begin{array}{l}\text { Precio x unidad } \\
\text { (dólares) }\end{array}$ & $\begin{array}{l}\text { Monto } \\
\text { (dólares) }\end{array}$ & $\begin{array}{l}\text { Precio x unidad } \\
\text { (dólares) }\end{array}$ & $\begin{array}{l}\text { Monto } \\
\text { (dólares) }\end{array}$ \\
\hline \multirow{2}{*}{1} & Limpieza inicial & suma fija & 1 & 50.000 & 50.000 & 50.000 & 50.000 \\
2 & Escavar Tierra & yarda cúbica & 50.000 & 1,5 & 75.000 & 1,25 & 62.500 \\
3 & Escavar Roca & yarda cúbica & 25.000 & 3 & 75.000 & 3,5 & 87.500 \\
4 & Limpieza final & suma fija & 1 & 50.000 & 50.000 & 50.000 & 50.000 \\
\hline
\end{tabular}

Fuente: Adaptado en base a un ejemplo de Gates (1967).

Los pagos al contratista con y sin quantity error exploitation se encuentran en la Tabla 5. Si las estimaciones del contratista en cuanto a las cantidades necesarias para desarrollar el proyecto fueron las correctas, desbalancear la licitación generaría 15,000 dólares adicionales. 
Tabla 5: Pago de la Licitación

\begin{tabular}{|c|c|c|c|c|c|c|c|}
\hline \multirow[b]{3}{*}{ Ítem No } & \multirow[b]{3}{*}{ Descripción } & \multirow[b]{3}{*}{ Unidad } & \multirow[b]{3}{*}{$\begin{array}{l}\text { Cantidad } \\
\text { Realizada }\end{array}$} & \multirow{2}{*}{\multicolumn{2}{|c|}{ Pliego A }} & \multirow{2}{*}{\multicolumn{2}{|c|}{$\begin{array}{c}\text { Pliego B } \\
\text { Quantity Error Exploitation }\end{array}$}} \\
\hline & & & & & & & \\
\hline & & & & $\begin{array}{l}\text { Precio x unidad } \\
\text { (dólares) }\end{array}$ & $\begin{array}{l}\text { Monto } \\
\text { (dólares) }\end{array}$ & $\begin{array}{l}\text { Precio } \mathrm{x} \text { unidad } \\
\text { (dólares) }\end{array}$ & $\begin{array}{l}\text { Monto } \\
\text { (dólares) }\end{array}$ \\
\hline 1 & Limpieza inicial & suma fija & 1 & 50.000 & 50.000 & 50.000 & 50.000 \\
\hline 2 & Escavar Tierra & yarda cúbica & 30.000 & 1,5 & 45.000 & 1,25 & 37.500 \\
\hline 3 & Escavar Roca & yarda cúbica & 45.000 & 3 & 135.000 & 3,5 & 157.500 \\
\hline \multirow[t]{2}{*}{4} & Limpieza final & suma fija & 1 & 50.000 & 50.000 & 50.000 & 50.000 \\
\hline & & & & Total & 280.000 & & 295.000 \\
\hline
\end{tabular}

Fuente: Adaptado en base a un ejemplo de Gates (1967).

Los contratistas tienen incentivos a desbalancear los mark-ups debido a que los precios ofertados forman parte del contrato y son usados para la valuación de las distintas instancias de pagos y potenciales variaciones de cantidades. No resulta fácil para el MDOT diferenciar entre pliegos balanceados y no balanceados ya que se desconocen los costos que enfrentarán los contratistas. El MDOT provee las especificaciones y el diseño del proyecto y el contratista se compromete a cumplir con los mismos. Pero los contratistas tienen total libertad para decidir su estrategia y técnicas para llevar a cabo el proyecto. La heterogeneidad de costos también se debe a que los contratistas pueden tener diferentes recursos disponibles, proveedores y subcontratistas.

\section{Base de Datos y Estadísticas Descriptivas}

Se utilizan los contratos de construcción ofrecidos vía subasta pública del departamento de transporte de Michigan para el período 2001-2013 $3^{3}$ La base de datos tiene información de i) la estimación total y por componentes del costo del proyecto del ingeniero, el pliego de la empresa ganadora y de las empresas que perdieron, ii) costo total realizado y por componentes de la empresa ganadora y iii) pagos realizados a la empresa ganadora en distintos períodos de tiempo. Los contratos incluyen diferentes tipo de proyectos: repavimentación del asfalto, pavimentación de rutas y reconstrucción de puentes entre otras categorías. Las unidades de análisis se detallan en la Tabla 6:

Tabla 6: Estadísticas generales

\begin{tabular}{lr}
\hline & Obs \\
\hline Contratistas & 624 \\
Contratos & 3,391 \\
Pliegos & 17,969 \\
Ítems & 24,148 \\
\hline
\end{tabular}

Fuente: Elaboración propia en base a licitaciones disponibles online.

\footnotetext{
${ }^{3}$ Los datos se encuentran disponibles en la página web del MDOT: http://mdotcf.state.mi.us/public/trnsport/.
} 
Antes de comenzar el análisis a nivel del ítem es importante contextualizar y presentar las estadísticas a nivel del pliego. En la Tabla 7 se presentan las estadísticas generales de los pliegos de los contratistas ${ }^{4}$. El monto promedio del pliego ganador es de $\$ 849,449$, el cual es un $7.4 \%$ menor que el estimado por el ingeniero del proyecto. Esta diferencia se debe a que el ingeniero y el contratista eligen sus precios basandose en distintas consideraciones. Por un lado, el ingeniero utiliza unos precios tabulados en los manuales de procedimientos del departamento de transporte que se actualizan anualmente y adiciona un $10 \%$ al total en concepto de gastos de movilidad y transporte. Y por el otro, el contratista elige el monto total de pliego para maximizar sus beneficios en base a las caracteristicas del mercado al momento de realizar el proyecto. El monto obtenido luego de realizar el proyecto es un $9.72 \%$ menor que el estimado por el ingeniero. Esta diferencia se debe a que, en promedio, las cantidades finales fueron menores a las estimadas por el ingeniero. Como el presupuesto del proyecto tiene que aprobarse antes de su licitación, en este caso, el ingeniero puede estar sobre-estimando las cantidades de manera sistemática para evitar excesos presupuestarios luego de la ejecución del proyecto.

Finalmente, el promedio de contratistas que presentan sus ofertas en una licitación es 5.3. La diferencia promedio en el monto del pliego entre el contratista ganador y el posicionado en segundo lugar es de \$50,441 o $7 \%$ del estimado (money left on the table). Esto significa que, en promedio, el ganador podría haber incrementado su oferta en ese monto y aún así ganar la licitación. La literatura empírica acerca de subastas interpreta este monto como evidencia de información asimétrica o incompleta.

Tabla 7: Estadísticas descriptivas de los pliegos

\begin{tabular}{lrrrrr}
\hline & Obs. & Media & Des. Est. & Min. & Max. \\
\hline Pliego Ganador $(\$)$ & 3391 & 849,449 & $1,364,588$ & 8,069 & $40,500,000$ \\
Mark-up: (Pliego Ganador-Estimado)/Estimado & 3391 & -0.0742 & 0.1514 & -0.7488 & 1.1569 \\
Pliego Normalizado: Pliego Ganador/Estimado & 3391 & 0.9258 & 0.1514 & 0.2512 & 2.1569 \\
Ex Post Mark-up:(Ingreso-Estimado)/Estimado & 3391 & -0.0972 & 0.1733 & -0.9365 & 2.0490 \\
Money on the Table (\$): $2^{\circ}$ Pliego-1 ${ }^{\circ}$ Pliego & 3327 & 50,441 & 103,701 & 0 & $1,852,379$ \\
Money on the Table Normalizado: $\left(2^{\circ}\right.$ Pliego-1 $1^{\circ}$ Pliego)/Estimado & 3327 & 0.0698 & 0.1229 & 0 & 5.3216 \\
Contratistas por contrato & 3391 & 5.30 & 3.28 & 1 & 23 \\
\hline
\end{tabular}

Fuente: Elaboración propia en base a licitaciones disponibles online.

Por último, como puede verse en la Tabla 8, la participación de los contratistas no es homogénea. Un gran porcentaje de los pliegos ofertados están concentrados en pocas firmas. Hay 46 contratistas que participaron en 100 o más licitaciones. Estos contratistas ofrecieron en total 9,715 pliegos y ganaron 1,933 licitaciones. Esto significa que el 57\% de los contratos están en manos de 7.4\% de los contratistas que alguna vez participaron. Por otro lado, hay 379 (60.7\%) contratistas que participaron entre 1 y 10 veces, en total ofrecieron 1,166 pliegos y ganaron 175 (5.2\%) licitaciones.

\footnotetext{
${ }^{4}$ Las estadísticas de los pliegos según nivel de participación se encuentran en la Tabla A1 del Anexo.
} 
Tabla 8: Cantidad de contratistas y licitaciones según participación

\begin{tabular}{rcccc}
\hline & & \multicolumn{3}{c}{ Pliegos } \\
\cline { 3 - 5 } Participación $^{*}$ & Contratistas & Presentados & Ganados & Ganados por contratista \\
\hline$[1-10]$ & 379 & 1,166 & 175 & 0.7 \\
{$[11-50]$} & 152 & 3,626 & 595 & 4.6 \\
{$[51-100]$} & 47 & 3,462 & 688 & 15.5 \\
{$[+100]$} & 46 & 9,715 & 1,933 & 62.8 \\
\hline Total & 624 & 17,969 & 3,391 & 37.9 \\
\hline
\end{tabular}

Fuente: Elaboración propia en base a licitaciones disponibles online.

Nota: ${ }^{*}$ Generada en base a la cantidad de pliegos presentados por firma.

\section{Modelo - Contratistas neutrales al riesgo}

En esta sección se desarrolla un modelo que considera que los contratistas obtienen su vector de precios óptimo dado unas creencias o información acerca de los ajustes de cantidades y supone que los contratistas son neutrales al riesgo.

El proyecto está caracterizado por tareas $j=1, \ldots, J$, y cantidades estimadas por el ingeniero para cada tarea $Q_{j}$. Sea $E=\sum_{j=1}^{J} Q_{j} p_{j}$ el monto total del proyecto estimado por el ingeniero del MDOT, donde $p_{j}$ son los valores de referencia unitarios del ingeniero. El contratista ofrece un vector de precios $b=\left(b_{1}, b_{2}, \ldots, b_{J}\right)$, donde $b_{j}$ es el precio unitario de la tarea $j$. El monto total del pliego es: $B=\sum_{j=1}^{J} Q_{j} b_{j}$. Sean $c_{j}$ los costos del contratista asociados a la tarea $j$.

El proyecto se completa en una cantidad de meses $T$ siendo $t$ un mes determinado. El departamento de transporte pagará a los contratistas a medida que vayan completando las tareas. Sea $\theta_{t j}$ la proporción que debe realizarse en el mes $t\left(\sum_{t=1}^{T} \theta_{t j}=1\right)$ y $r$ la tasa de descuento mensual. El valor presente de los pagos para la tarea $j$ será igual al valor nominal multiplicado por el factor de descuento: $V_{j}^{e}=\sum_{t=1}^{T}\left(\frac{1}{1+r}\right)^{t} \theta_{t j}$.

La cantidad que se termina realizando para cada tarea puede ser diferente a la originalmente estimada por el ingeniero del proyecto. Los contratistas poseen sus propias predicciones en cuanto al verdadero monto necesario para cada tarea, el cual puede coincidir o no con el del ingeniero. Sea $\Delta Q_{j}^{e}$ la variación de la cantidad estimada por el contratista de la tarea $j$. Entonces, el valor presente de los flujos de ingreso del contratista va a ser igual al valor presente de las cantidades estimadas por el ingeniero más el valor presente de la variación de la cantidad estimada por el contratista: $\left(V_{j}^{e} Q_{j}+V_{j}^{e} \Delta Q_{j}^{e}\right)$.

El problema del contratista es elegir el monto total del pliego $\bar{B}$ y la asignación de precios entre los componentes del proyecto $\left\{b_{j}\right\}_{j=1}^{J}$ para maximizar sus beneficios esperados sin desbalancear el pliego excesivamente. Formalmente, se maximiza el valor presente de los beneficios esperados (1) sujeto a: (2) que el monto total del pliego sea igual a un monto determinado $\bar{B}$ y (3) que los precios 
estén acotados a un cierto nivel para no correr el riesgo de ser detectado y descalificado.

$$
\begin{aligned}
& \operatorname{Max}_{\bar{B},\left\{b_{j}\right\}}\left(\sum_{j=1}^{J}\left[V_{j}^{e} Q_{j}+V_{j}^{e} \Delta Q_{j}^{e}\right] b_{j}-\sum_{j=1}^{J} c_{j} Q_{j}^{e}\right) P(\text { Ganar } \mid \bar{B}) \\
& \text { sujeto } a \text { : } \\
& \sum_{j=1}^{J} Q_{j} b_{j}=\bar{B} \\
& \underline{b}_{j} \leq b_{j} \leq \bar{b}_{j}
\end{aligned}
$$

donde $P($ Ganar $\mid \bar{B})$ es la probabilidad de ganar dado un $\bar{B}$ determinado. Si los contratistas son neutrales al riesgo, es posible separar este problema en dos partes:

1. La elección del monto total a ofertar: el contratista debe elegir $\bar{B}$ de manera tal que pueda ganar con una probabilidad suficientemente alta y obtener beneficios en caso de ganar. Formalmente,

$$
\operatorname{Max}_{\{\bar{B}\}}\left(V\left(\bar{B}, Q^{e}\right)-\sum_{j=1}^{J} c_{j} Q_{j}^{e}\right) P(\text { Ganar } \mid \bar{B})
$$

donde $V\left(\bar{B}, Q^{e}\right)$ es el máximo ingreso esperado que el contratista puede generar con un monto total $\bar{B}$ y unas cantidades esperadas $Q^{e}$.

2. La asignación de precios unitarios a cada tarea: el contratista debe asignar los precios unitarios a cada tarea que le permitan maximizar su ingreso esperado. Formalmente, se soluciona el siguiente problema lineal:

$$
\begin{aligned}
& V\left(\bar{B}, Q^{e}\right)=\operatorname{Max}_{\left\{b_{j}\right\}} \sum_{j=1}^{J}\left[V_{j}^{e} Q_{j}+V_{j}^{e} \Delta Q_{j}^{e}\right] b_{j} \\
& \text { sujeto } a \text { : } \\
& \sum_{j=1}^{J} Q_{j} b_{j}=\bar{B} \\
& \underline{b}_{j} \leq b_{j} \leq \bar{b}_{j}
\end{aligned}
$$

Si las tareas se ordenan de forma que el término $\left[V_{j}^{e}+V_{j}^{e} \frac{\Delta Q_{j}^{e}}{Q_{j}}\right]$ es decreciente en $j$, el problema lineal tiene una solución en la que $\forall j>j^{*}$ el precio asignado es igual a su cota mayor $b_{j}=\bar{b}_{j}$, $\forall j<j^{*}$ el precio asignado es igual a su cota menor $b_{j}=\underline{b}_{j}$ y $b_{j^{*}}$ toma un valor tal que la restricción de igualdad $(B=\bar{B})$ se cumpla.

Como puede verse en (4), los precios unitarios óptimos que maximizan los ingresos esperados no dependen de los costos del contratista. Esto es una consecuencia del supuesto de neutralidad al riesgo que permite separar el problema del contratista en dos partes. Este trabajo sólo se focaliza 
en el problema de la elección óptima de los precios dado un nivel $\bar{B}$ determinado. La elección de $\bar{B}$ no es relevante para los propósitos de este trabajo.

Las variables exógenas para el contratista y el econometrista son: las estimaciones del ingeniero del MDOT respecto a cantidades $\left(Q_{j}\right)$, los precios por unidad $\left(p_{j}\right)$, el monto total del proyecto $(E)$, la proporción de $Q_{j}$ que se realiza en el mes $t\left(\theta_{t j}\right)$ y el monto total del pliego $(B)$. El econometrista también observa las variaciones de las cantidades realizadas $\left(\Delta Q_{j}\right)$, mientras que al momento de preparar el pliego el contrastista sólo tiene una predicción $\left(\Delta Q_{j}^{e}\right)$. Las variables observables para el contratista pero no para el econometrista son: las predicciones $\left(\Delta Q_{j}^{e}\right)$, la tasa de descuento mensual $(r)$ y los límites a los precios. Estas tres variables serán consideradas errores estructurales. Con esta información el contratista decide los precios unitarios ofertados $\left(b_{j}\right)$ los cuales son observados para el econometrista.

\subsection{Cálculo de las rentas de desbalanceo}

En esta sección se desarrolla el cálculo de la renta que permite distinguir las estrategias de front-end loading y/o quantity error exploitation por separado.

Supongamos que el vector de precios que re-escala los valores estimados por el ingeniero $\left\{p_{j}\right\}$ es factible. Es decir, los contratistas pueden utilizar este vector de precios sin ser descalificados. Formalmente, se define el factor $\alpha=\frac{B}{E}$ y se supone que $\underline{b}_{j} \leq \alpha p_{j} \leq \bar{b}_{j}$. Si el contratista está utilizando la información a su favor, la solución al problema (4) debe otorgarle mayores ingresos esperados que lo que le proporciona el vector de precios re-escalado $\left\{\alpha p_{j}\right\}$. Sea $\left\{b_{j}\right\}$ la solución al problema:

$$
\sum_{j=1}^{J}\left[V_{j}^{e} Q_{j}+V_{j}^{e} \Delta Q_{j}^{e}\right] b_{j}>\sum_{j=1}^{J}\left[V_{j}^{e} Q_{j}+V_{j}^{e} \Delta Q_{j}^{e}\right] \alpha p_{j}
$$

Reemplazando $\alpha$ por $\frac{B}{E}=\frac{\sum_{j=1}^{J} Q_{j} b_{j}}{\sum_{j=1}^{J} Q_{j} p_{j}}$ y reordenando:

$$
\frac{\sum_{j=1}^{J}\left[V_{j}^{e} Q_{j}+V_{j}^{e} \Delta Q_{j}^{e}\right] b_{j}}{\sum_{j=1}^{J} Q_{j} b_{j}}>\frac{\sum_{j=1}^{J}\left[V_{j}^{e} Q_{j}+V_{j}^{e} \Delta Q_{j}^{e}\right] p_{j}}{\sum_{j=1}^{J} Q_{j} p_{j}}
$$

Intuitivamente, el contratista asignará los precios a cada una de las tareas de manera tal que el incremento porcentual en el pago esperado utilizando los precios ofrecidos en el pliego sea mayor que el obtenido utilizando los valores del ingeniero. Reordenando la ecuación:

$$
\sum_{j=1}^{J}\left[\frac{b_{j}}{\sum_{j=1}^{J} Q_{j} b_{j}}-\frac{p_{j}}{\sum_{j=1}^{J} Q_{j} p_{j}}\right]\left[V_{j}^{e} Q_{j}+V_{j}^{e} \Delta Q_{j}^{e}\right]>0
$$

Multiplicando y dividiendo por $Q_{j}$ :

$$
\begin{gathered}
\sum_{j=1}^{J}\left[\frac{Q_{j} b_{j}}{\sum_{j=1}^{J} Q_{j} b_{j}}-\frac{Q_{j} p_{j}}{\sum_{j=1}^{J} Q_{j} p_{j}}\right]\left[V_{j}^{e}+V_{j}^{e} \frac{\Delta Q_{j}^{e}}{Q_{j}}\right]>0 \\
\sum_{j=1}^{J}\left[\tilde{b}_{j}-\tilde{p}_{j}\right]\left[V_{j}^{e}+V_{j}^{e} \Delta \tilde{q}_{j}^{e}\right]>0
\end{gathered}
$$


donde $\tilde{b}_{j}=\frac{Q_{j} b_{j}}{\sum_{j=1}^{J} Q_{j} b_{j}}$ es la participación de cada componente en el pliego, $\tilde{p}_{j}=\frac{Q_{j} p_{j}}{\sum_{j=1}^{J} Q_{j} p_{j}}$ es la participación de cada componente en el costo total del ingeniero y $\Delta \tilde{q}_{j}^{e}=\frac{\Delta Q_{j}^{e}}{Q_{j}}$ es la variación porcentual esperada de las cantidades. Reordenando la ecuación se obtiene la renta de los contratistas:

$$
R=\sum_{j=1}^{J}\left(\tilde{b}_{j}-\tilde{p}_{j}\right) V_{j}^{e}+\left(\tilde{b}_{j}-\tilde{p}_{j}\right) V_{j}^{e} \Delta \tilde{q}_{j}^{e}>0
$$

Entonces, si $R>0$ existe front-end loading y/o quantity error exploitation. En particular, es posible obtener la contribución de cada uno de los componentes. Front-end loading está asociado al término $\sum_{j=1}^{J}\left(\tilde{b}_{j}-\tilde{p}_{j}\right) V_{j}^{e}$, mientras que $\sum_{j=1}^{J}\left(\tilde{b}_{j}-\tilde{p}_{j}\right) V_{j}^{e} \Delta \tilde{q}_{j}^{e}$ está asociado a quantity error explotation.

Las tareas que están programadas para realizarse al principio del proyecto van a tener valores altos de $V_{j}^{e}$. Por lo tanto, las mismas van a generar un valor presente del pago más elevado que la misma tarea programada más tarde en el proyecto. A su vez, cuanto mayor sea la tasa de descuento $r$ mayor será el diferencial de $V_{j}^{e}$ entre tareas cuyos pagos están programados al principio y al final del proyecto. Esto corresponde con el principio de front-end loading en el cual se reconoce que si se asignan precios más elevados a aquellas tareas programadas al principio del proyecto, las mismas van generar mayores ingresos que aquellas tareas programadas más tarde y con precios similares.

Las tareas que tienen un valor alto de $\Delta Q_{j}^{e}$ van a generar pagos más elevados, especialmente cuando se combina con la asignación de $b_{j}$ más elevados. El principio de quantity error exploitation asigna precios más elevados a aquellas tareas que se creen que están subestimadas (y viceversa).

A continuación, se testea empíricamente la existencia de front-end loading y de quantity error exploitation. Para esta última estrategia, se realizan 3 contraste de hipótesis acerca de la información que tiene el contratista respecto a la variación futura de las cantidades: i) los contratistas no tienen información más precisa que el ingeniero, ii) los contratistas no tienen información mas precisa que el consultor, y iii) los contratistas tienen la misma información. En la primera hipótesis se comparan los retornos que los contratistas efectivamente obtienen con los retornos que obtendrían si no tuvieran ningún tipo de información acerca de la variación futura de las cantidades. En este ejercicio, el vector de precios contrafactual es el que simplemente re-escala los valores del ingeniero. En la segunda hipótesis se comparan los retornos que los contratistas obtienen en los datos con los retornos que obtendrían si conocieran la distribución empírica de los ajustes de cantidades al momento de presentar sus ofertas. En este caso, el vector de precios contrafactual o del consultor se obtiene de resolver el mismo problema de maximización que en (4) pero con la variante que se utiliza la distribución empírica de los ajustes de cantidades en su cálculo. Por último, en la tercera hipótesis se analiza si los contratistas ganadores tienen la misma información. Para poder determinar la heterogeneidad de la información entre las firmas se realiza un análisis de regresión y se comparan las firmas bajo los contrate de hipótesis i) y ii). 


\subsection{Evidencia de Front-End loading}

Para verificar la existencia de front-end loading se testea su contribución en la renta del contratista. De la ecuación (5) resulta:

$$
R_{f l}=\sum_{j=1}^{J}\left(\tilde{b}_{j}-\tilde{p}_{j}\right) V_{j}^{e}(r)>0
$$

Por construcción, la renta va a ser mayor cuanto menor sea la tasa de descuento. Para eliminar este efecto se la normaliza dividiendola por el factor: $\sum_{j=1}^{J} \frac{V_{j}^{e}(r)}{V_{j}^{e}(0)}$. Dado que no se conoce la tasa de descuento mensual $(r)$ que utilizan los contratistas, se considerarán distintos escenarios en los cuales se calculan los pagos del ítem $j$ a diferentes tasas de descuento.

La renta $R_{f l}$ resulta ser la fórmula de la covarianza entre $V_{j}^{e}(r)$ y $\left(\tilde{b}_{j}-\tilde{p}_{j}\right)$. El componente $V_{j}^{e}(r)$ es alto para ítems que se pagan al comienzo del proyecto y bajo para ítems que se pagan al final. Si los contratistas están haciendo front-end loading, eligen $\left(\tilde{b}_{j}-\tilde{p}_{j}\right)$ altos para los ítems que se pagan al comienzo introduciendo una covarianza positiva con $V_{j}^{e}(r)$. En cambio, si los contratistas no están haciendo front-end loading, la renta $R_{f l}$ va a ser cero o negativa porque $\left(\tilde{b}_{j}-\tilde{p}_{j}\right)$ no está correlacionado positivamente con $V_{j}^{e}(r)$.

Los resultados para distintas tasas de descuento se encuentran en la Tabla 9. No existe evidencia estadísticamente significativa de que los contratistas estén realizando front-end loading sistemáticamente. Como puede verse en los valores medios y medianos de $R_{f l}$, su distribución se encuentra centrada en cero independientemente de la tasa de descuento. A su vez, la varianza de $R_{f l}$ aumenta a medida que se consideran tasas de descuento más elevadas y esto también se refleja en los distintos percentiles. Por lo tanto, de ahora en adelante, en este trabajo se analizará solamente el efecto de quantity error exploitation.

Tabla 9: Evidencia de Front End- Loading

\begin{tabular}{|c|c|c|c|c|c|c|c|c|c|c|}
\hline \multirow{2}{*}{$\begin{array}{l}\text { Tasa de } \\
\text { Descuento }\end{array}$} & \multirow[t]{2}{*}{ Obs. } & \multirow[t]{2}{*}{ Media } & \multirow[t]{2}{*}{ Des. Est. } & \multirow[t]{2}{*}{ T-stat } & \multicolumn{5}{|c|}{ Percentiles } & \multirow[t]{2}{*}{$\operatorname{Prob}\left(R_{f l}\right)>0$} \\
\hline & & & & & 5 & 25 & 50 & 75 & 95 & \\
\hline 0 & 3391 & 0.00000 & 0.00000 & 0.21 & 0.00000 & 0.00000 & 0.00000 & 0.00000 & 0.00000 & $49.9 \%$ \\
\hline 2 & 3391 & -0.00128 & 0.06303 & -1.18 & -0.00034 & -0.00007 & -0.00001 & 0.00004 & 0.00024 & $45.4 \%$ \\
\hline 4 & 3391 & -0.00130 & 0.06306 & -1.20 & -0.00067 & -0.00014 & -0.00001 & 0.00009 & 0.00048 & $45.4 \%$ \\
\hline 6 & 3391 & -0.00132 & 0.06307 & -1.22 & -0.00098 & -0.00021 & -0.00002 & 0.00013 & 0.00071 & $45.4 \%$ \\
\hline 8 & 3391 & -0.00134 & 0.06308 & -1.24 & -0.00128 & -0.00027 & -0.00002 & 0.00017 & 0.00093 & $45.5 \%$ \\
\hline 10 & 3391 & -0.00136 & 0.06309 & -1.25 & -0.00158 & -0.00034 & -0.00003 & 0.00021 & 0.00114 & $45.4 \%$ \\
\hline 12 & 3391 & -0.00137 & 0.06310 & -1.27 & -0.00186 & -0.00040 & -0.00003 & 0.00024 & 0.00135 & $45.4 \%$ \\
\hline 14 & 3391 & -0.00139 & 0.06310 & -1.28 & -0.00213 & -0.00046 & -0.00004 & 0.00028 & 0.00155 & $45.4 \%$ \\
\hline 16 & 3391 & -0.00141 & 0.06311 & -1.30 & -0.00240 & -0.00052 & -0.00004 & 0.00032 & 0.00175 & $45.4 \%$ \\
\hline 18 & 3391 & -0.00142 & 0.06312 & -1.31 & -0.00267 & -0.00058 & -0.00005 & 0.00035 & 0.00194 & $45.4 \%$ \\
\hline 20 & 3391 & -0.00144 & 0.06313 & -1.33 & -0.00292 & -0.00063 & -0.00005 & 0.00039 & 0.00211 & $45.3 \%$ \\
\hline
\end{tabular}

Fuente: Elaboración propia en base a licitaciones disponibles online.

Nota: p-values ${ }^{*} \mathrm{p}<0.1,{ }^{*} * \mathrm{p}<0.05,{ }^{* * *} \mathrm{p}<0.01$ 


\section{3. ¿Los contratistas tienen información más precisa que el ingeniero?}

La primera hipótesis a testear es si, en promedio, los contratistas no obtienen mayores ingresos que los que hubieran obtenido si elegían el vector de precios del ingeniero re-escalado. En otras palabras, se compara los retornos que los contratistas efectivamente obtienen con los retornos que obtendrían si no tuvieran ningún tipo de información acerca de la variación futura de las cantidades. Esto significa que: i) las expectativas que tiene el contratista respecto a la variación porcentual esperada de las cantidades $\left(\Delta \tilde{q}^{e}\right)$ no predicen a las cantidades realizadas, y ii) el contratista no sesga en forma positiva y sistemática los ítems que en promedio aumentan. En otras palabras, el contratista no conoce la distribución de los ajustes de cantidades.

Un contratista podría tomar el informe del ingeniero y re-escalar cada uno de sus precios unitarios proporcionalmente. Si tiene algún tipo de información que le permita predecir que un ítem va a ser modificado, puede sesgar su pliego en la dirección correspondiente. A pesar de que no se observa la información que tienen los contratistas al momento de realizar sus ofertas, se puede inferir la calidad de la misma comparando los ingresos que obtienen en los datos con los ingresos que obtendrían si simplemente re-escalan los precios del ingeniero. Entonces, si los contratistas obtienen mayores retornos, se concluye que los mismos tienen información más precisa que el ingeniero y actúan sobre ella desbalanceando sus pliegos (o haciendo bid skewing). Y si obtienen retornos iguales o inferiores, se concluye que los contratistas no tienen información más precisa que el ingeniero ${ }^{5}$.

A partir de la ecuación (5) se obtiene la contribución del quantity error exploitation en la renta del contratista:

$$
R_{q e}=\sum_{j=1}^{J}\left(\tilde{b}_{j}-\tilde{p}_{j}\right)\left(1+\Delta \tilde{q}_{j}^{f}\right)>0
$$

donde $\Delta \tilde{q}_{j}^{f}$ es la variación porcentual de las cantidades realizada o final. De esta manera, es posible comparar el monto final que resulta de utilizar los precios unitarios observados contra los precios proporcionales a los del ingeniero.

En promedio, el monto obtenido utilizando los precios observados es mayor. Los contratistas ganadores obtienen un $1.6 \%$ adicional. A simple vista, este monto pareciera ser muy pequeño, sin embargo, resulta ser significativo cuando se lo analiza en términos de los mark-ups. Por ejemplo, supongamos que el contratista fija un mark-up para el proyecto del $10 \%$. En este caso, el beneficio del contratista ganador se incrementaría, en promedio, en un $16 \%$ debido a la estrategia de quantity error exploitation.

También es posible calcular cuánto hubiera sido el ingreso de los contratistas que no ganaron el proyecto y realizar la misma comparación. Los ubicados en segundo lugar hubieran obtenido un $1.3 \%$ adicional por sobre lo que les correspondería en el caso que hubiesen utilizado precios unitarios proporcionales a los del ingeniero.

\footnotetext{
${ }^{5} \mathrm{Si}$ los contratistas tienen mayor información y no actúan sobre ella, voy a considerarlo como si los contratistas nunca tuvieron esa información.
} 
Para poder verificar la robustez de estos resultados, se calcula la renta para distintos puntos de la distribución. Como puede verse en la Tabla 10, la mediana, a pesar de ser menor que los valores promedio, sigue siendo positiva y decreciente con el ranking. Además, en el $64.1 \%$ de las licitaciones el contratista ganador utilizó un vector de precios que le aseguró un ingreso mayor al vector re-escalado del ingeniero.

Tabla 10: Evidencia de Quantity Error Exploitation

\begin{tabular}{|c|c|c|c|c|c|c|c|c|c|c|}
\hline \multirow[t]{2}{*}{ Rank } & \multirow[t]{2}{*}{ Obs. } & \multirow[t]{2}{*}{ Media } & \multirow[t]{2}{*}{ Des. Est. } & \multirow[t]{2}{*}{ T-stat } & \multicolumn{5}{|c|}{ Percentiles } & \multirow[t]{2}{*}{$\operatorname{Prob}\left(R_{q e}\right)>0$} \\
\hline & & & & & 5 & 25 & 50 & 75 & 95 & \\
\hline 1 & 3391 & 0.0159 & 0.0531 & $17.5^{* * *}$ & -0.0324 & -0.0042 & 0.0060 & 0.0261 & 0.0924 & $64.1 \%$ \\
\hline 2 & 3328 & 0.0134 & 0.0519 & $14.9^{* * *}$ & -0.0369 & -0.0050 & 0.0045 & 0.0227 & 0.0878 & $63.1 \%$ \\
\hline 3 & 2818 & 0.0120 & 0.0476 & $13.4^{* * *}$ & -0.0366 & -0.0054 & 0.0049 & 0.0226 & 0.0860 & $62.1 \%$ \\
\hline
\end{tabular}

Fuente: Elaboración propia en base a licitaciones disponibles online.

Nota: $\mathrm{p}$-values ${ }^{*} \mathrm{p}<0.1,{ }^{* *} \mathrm{p}<0.05, * * * \mathrm{p}<0.01$

Por último, en esta sección se analiza cuál es el grupo de contratistas ganadores, definidos según la cantidad de pliegos presentados por firma, que utiliza vectores de precios que le permite obtener mayores ingresos. El objetivo es determinar si la mayor participación del contratista en este mercado de licitaciones está correlacionada con mayor información.

Como puede verse en la Tabla 11, la media y la mediana de $R_{q e}$ son positivas y estadísticamente significativas para todos los grupos. Además, la magnitud de la renta es creciente con el nivel de participación. En particular, el grupo con mayor participación obtiene un 1.9\% de ingreso adicional respecto a los precios re-escalados del ingeniero. Esto significa que los contratistas que participan más seguido parecen tener información más precisa respecto a la variación de las cantidades y obtienen mayores beneficios realizando bid skewing ${ }^{6}$.

Tabla 11: Evidencia de Quantity Error Exploitation según participación

\begin{tabular}{|c|c|c|c|c|c|c|c|c|c|c|}
\hline \multirow[t]{2}{*}{ Participación ${ }^{*}$} & \multirow[t]{2}{*}{ Obs. } & \multirow[t]{2}{*}{ Media } & \multirow[t]{2}{*}{ Des. Est. } & \multirow[t]{2}{*}{ T-stat } & \multicolumn{5}{|c|}{ Percentiles } & \multirow[t]{2}{*}{$\operatorname{Prob}\left(R_{q e}\right)>0$} \\
\hline & & & & & 5 & 25 & 50 & 75 & 95 & \\
\hline$[1-10]$ & 175 & 0.0084 & 0.0437 & $2.5^{* * *}$ & -0.0425 & -0.0058 & 0.0060 & 0.0238 & 0.0719 & $63.4 \%$ \\
\hline$[11-50]$ & 595 & 0.0098 & 0.0420 & $5.7^{* * * *}$ & -0.0405 & -0.0067 & 0.0021 & 0.0195 & 0.0885 & $58.3 \%$ \\
\hline$[51-100]$ & 688 & 0.0139 & 0.0424 & $8.6^{* * *}$ & -0.0300 & -0.0032 & 0.0066 & 0.0244 & 0.0898 & $65.4 \%$ \\
\hline$[+100]$ & 1933 & 0.0193 & 0.0598 & $14.2^{* * *}$ & -0.0299 & -0.0040 & 0.0070 & 0.0302 & 0.0987 & $65.5 \%$ \\
\hline
\end{tabular}

Fuente: Elaboración propia en base a licitaciones disponibles online.

Nota: $\mathrm{p}$-values $* \mathrm{p}<0.1, * * \mathrm{p}<0.05, * * * \mathrm{p}<0.01$

\footnotetext{
${ }^{6}$ Este trabajo utiliza los términos "desbalanceo estratégico de precios en las licitaciones" y bid skewing en forma indistinta.
} 
En conclusión, de esta sección se confirma que, en promedio, los contratistas están realizando bid skewing y el mismo viene explicado por la práctica de quantity error exploitation. Es decir, existen asimetrías de la información entre los contratistas y el licitador.

También existen asimetrías de la información ente los contratistas. Los contratistas ganadores son quienes obtienen mayores ingresos haciendo bid skewing. A su vez, los contratistas ganadores con mayor participación son quienes obtienen mayores beneficios lo cual se interpreta como una ventaja informativa ${ }^{7}$.

\section{4. ¿Los contratistas tienen información más precisa que el consultor?}

La segunda hipótesis que resulta interesante testear es si, en promedio, los contratistas tienen información más precisa que la contenida en la distribución empírica de los ajustes de cantidades. Es decir, en esta sección se comparan los retornos que los contratistas efectivamente obtienen con los retornos que obtendrían si supieran la distribución observada en los datos.

Para la construcción del vector de precios contrafactual, se supone que existe un consultor que tiene acceso a una base de datos con información acerca de las licitaciones ofertadas en un período determinado. La misma incluye los precios ofertados por los contratistas y los del ingeniero para cada uno de los componentes del proyecto, y las cantidades estimadas y realizadas. Es decir, la misma información que se usa en este trabajo.

El consultor puede asesorar al contratista estimando las variaciones porcentuales de las cantidades finales $\left(\Delta \tilde{q}_{j}^{f}\right)$ y los límites posibles $\left(\underline{b}_{j}\right.$ y $\left.\bar{b}_{j}\right)$ :

1. La variación porcentual promedio de las cantidades para el ítem $j$ se estima calculando $\bar{q}_{j}=$ $\frac{1}{A} \sum_{a=1}^{A} \Delta \tilde{q}_{a j}^{f}$ donde $a=1, \ldots, A$ indexa los proyectos que contienen ese ítem. Se ordenan las $\bar{q}_{j}$ de manera tal que $\bar{q}_{j}>\ldots>\bar{q}_{k} \forall j \neq k$.

2. Para calcular los precios límites $\underline{b}_{j}$ y $\bar{b}_{j}$ y que no despierten sospechas, el consultor puede utilizar el comportamiento observado de los contratistas. Se calcula el índice $\pi_{i j}=\frac{b_{i j}}{p_{i j}} \frac{E_{i}}{B_{i}}$ que representa el desvío de los precios ofertados respecto a los del ingeniero re-escalado por los montos ofertados totales y estimados. Para cada ítem $j$, se calculan los percentiles $p_{(\tau)}\left[\pi_{j}\right]$ y $p_{(1-\tau)}\left[\pi_{j}\right]$ de la distribución empírica del índice $\pi_{i j}$ (la variación proviene de observar la misma tarea en diversos proyectos).

Entonces, ¿cómo asignaría el consultor los precios si tuviera que asesorar al contratista y éste sólo le provee información del monto total del pliego a ofertar? El consultor maximizará los ingresos esperados $^{8}$ sujeto a: i) el monto total del pliego sea igual al monto determinado por el contratista; y ii) los precios son acotados de manera tal que: $\underline{b}_{j}=\frac{B}{E} p_{(\tau)}\left[\pi_{j}\right] p_{j}$ y $\bar{b}_{j}=\frac{B}{E} p_{(1-\tau)}\left[\pi_{j}\right] p_{j}$.

\footnotetext{
${ }^{7}$ Las Tablas A2 y A3 del Anexo presentan los resultados a nivel del ítem.

${ }^{8}$ En la sección anterior se demostró que no hay evidencia estadísticamente significativa de que los contratistas estén haciendo front-loading $\left(V_{j}^{e}=1\right)$.
} 
Formalmente,

$$
\begin{aligned}
& \operatorname{Max}_{\left\{\hat{b}_{j}\right\}} \sum_{j=1}^{J} Q_{j}\left(1+\bar{q}_{j}\right) \hat{b}_{j} \\
& \text { sujeto } a: \\
& \sum_{j=1}^{J} Q_{j} \hat{b}_{j}=\bar{B} \\
& \underline{b}_{j} \leq \hat{b}_{j} \leq \bar{b}_{j}
\end{aligned}
$$

El problema lineal tiene una solución en la que $\forall j>j^{*}$ el precio asignado es igual a su cota mayor $\hat{b}_{j}=\bar{b}_{j}, \forall j<j^{*}$ el precio asignado es igual a su cota menor $\hat{b}_{j}=\underline{b}_{j}$ y $\hat{b}_{j^{*}}$ toma un valor tal que la restricción de igualdad $(B=\bar{B})$ se cumpla.

Entonces, si el contratista tiene información más precisa que el consultor (realizando los mismos cálculos matemáticos que en la sección 4.1), resulta:

$$
R_{q e}=\sum_{j=1}^{J}\left(\tilde{b}_{j}-\tilde{d}_{j}\right)\left(1+\Delta \tilde{q}_{j}^{f}\right)>0
$$

donde $\tilde{d}_{j}=\frac{Q_{j} d_{j}}{\sum_{j=1}^{J} Q_{j} d_{j}}$ es la participación de cada componente en el monto total del contratista utilizando el vector de precios del consultor.

Para esta base de datos en particular se utilizan 317 descripciones presentes en al menos 100 contratos y cuyas unidades no son de suma fija (las mismas representan $54.10 \%$ de las descripciones o ítems de la base). La variable $\bar{q}_{j}$ se truncó entre los límites 0 y 2 (10\% de las observaciones son mayores a 2). La presencia de valores extremos en la mayoría de las descripciones tiene mucha influencia en los resultados y se decidió truncarlos. La tabla A4 presenta las 25 descripciones con mayor frecuencia con sus respectivas $\bar{q}_{j}, p_{(10)}\left[\pi_{j}\right]$ y $p_{(90)}\left[\pi_{j}\right]$.

Los resultados del segundo contraste de hipótesis se encuentran en las Tablas 12 y 13 . La media de $R_{q e}$ solamente es positiva para el grupo ganador pero no es estadísticamente significativa; y la mediana es negativa y cercana a cero para todos los rankings. Estos resultados indican que los ingresos que obtienen los contratistas ganadores no son superiores a los que hubieran obtenido si utilizaban el vector de precios del consultor, y que los contratistas ubicados en segundo y tercer lugar obtienen ingresos menores a los que hubieran obtenido utilizando los precios del consultor. Esto significa que los contratistas parecen no tener información más precisa que la que tiene el consultor. En particular, los contratistas ubicados en segundo y tercer lugar parecen tener información menos precisa que el consultor.

Vale la pena destacar que la renta es sustancialmente menor que la obtenida comparando los precios del ingeniero para todos los rankings (Tabla 10). Por lo tanto, existe cierto beneficio para el ingeniero de utilizar información agregada de los ítems con mayor frecuencia al momento de calcular los precios y cantidades estimadas del proyecto. 
Tabla 12: Evidencia de Quantity Error Exploitation

\begin{tabular}{|c|c|c|c|c|c|c|c|c|c|c|}
\hline \multirow[t]{2}{*}{ Rank } & \multirow[t]{2}{*}{ Obs. } & \multirow[t]{2}{*}{ Media } & \multirow[t]{2}{*}{ Des. Est. } & \multirow[t]{2}{*}{ T-stat } & \multicolumn{5}{|c|}{ Percentiles } & \multirow[t]{2}{*}{$\operatorname{Prob}\left(R_{q e}\right)>0$} \\
\hline & & & & & 5 & 25 & 50 & 75 & 95 & \\
\hline 1 & 3391 & 0.0008 & 0.0671 & 0.7 & -0.0660 & -0.0179 & -0.0013 & 0.0142 & 0.0785 & $46.1 \%$ \\
\hline 2 & 3328 & -0.0017 & 0.0641 & $-1.5^{*}$ & -0.0710 & -0.0199 & -0.0025 & 0.0127 & 0.0725 & $43.3 \%$ \\
\hline 3 & 2818 & -0.0032 & 0.0551 & $-3.1^{* * *}$ & -0.0759 & -0.0221 & -0.0030 & 0.0120 & 0.0656 & $42.7 \%$ \\
\hline
\end{tabular}

Fuente: Elaboración propia en base a licitaciones disponibles online.

Nota: $\mathrm{p}$-values ${ }^{*} \mathrm{p}<0.1,{ }^{* *} \mathrm{p}<0.05,{ }^{* * *} \mathrm{p}<0.01$

A continuación se analiza a los contratistas ganadores que son los únicos que parecen tener información similar a la que posee el consultor. La Tabla 13 presenta la renta de los contratistas ganadores por grupos según participación. Sólo la renta media del grupo con mayor participación es positiva y estadísticamente significativa. Este grupo elige, en promedio, un vector de precios que le permite obtener ingresos $0.4 \%$ superiores a los que hubiera obtenido el consultor. Esto significa que el grupo con mayor participación es el único que parece tener información más precisa que el consultor. Sin embargo, la mediana de la renta es negativa lo cual indicaría que la distribución de la renta para este grupo es asimétrica. El percentil 95 de la distribución es 0.09 mientras que el quinto percentil es -0.06 indicando así que la cola derecha de la distribución es más larga. Esto amerita investigar con detalle qué está sucediendo en esta parte de la distribución. La próxima sección analiza si existen firmas que son más propensas a obtener rentas más altas.

Tabla 13: Evidencia de Quantity Error Exploitation según participación

\begin{tabular}{|c|c|c|c|c|c|c|c|c|c|c|}
\hline \multirow[t]{2}{*}{ Participación ${ }^{*}$} & \multirow[t]{2}{*}{ Obs. } & \multirow[t]{2}{*}{ Media } & \multirow[t]{2}{*}{ Des. Est. } & \multirow[t]{2}{*}{ T-stat } & \multicolumn{5}{|c|}{ Percentiles } & \multirow[t]{2}{*}{$\operatorname{Prob}\left(R_{q e}\right)>0$} \\
\hline & & & & & 5 & 25 & 50 & 75 & 95 & \\
\hline$[1-10]$ & 175 & -0.0015 & 0.0461 & -0.4 & -0.0812 & -0.0150 & 0.0000 & 0.0202 & 0.0670 & $50.3 \%$ \\
\hline$[11-50]$ & 595 & -0.0043 & 0.0488 & $-2.1^{* *}$ & -0.0718 & -0.0191 & -0.0021 & 0.0104 & 0.0650 & $41.7 \%$ \\
\hline$[51-100]$ & 688 & -0.0038 & 0.0767 & -1.3 & -0.0636 & -0.0154 & -0.0008 & 0.0120 & 0.0658 & $46.5 \%$ \\
\hline$[+100]$ & 1933 & 0.0041 & 0.0696 & $2.6 * * *$ & -0.0626 & -0.0184 & -0.0014 & 0.0159 & 0.0893 & $46.4 \%$ \\
\hline
\end{tabular}

Fuente: Elaboración propia en base a licitaciones disponibles online.

Nota: $\mathrm{p}$-values ${ }^{*} \mathrm{p}<0.1,{ }^{* *} \mathrm{p}<0.05, * * * \mathrm{p}<0.01$

\section{5. ¿Todos los contratistas ganadores tienen la misma información?}

Hasta aquí se aprendió que, en promedio, los contratistas ganadores tienen información más precisa que el ingeniero y similar a la del consultor. A su vez, los contratistas ganadores con mayor participación son quienes mayor bid skewing realizan y son los únicos que parecen tener información más precisa que el consultor.

Para terminar, la tercera hipótesis a contrastar es si existen ciertos contratistas con información más precisa que otros o es el grupo ganador en su conjunto el que tiene información más precisa. 
Para este ejercicio, se eligieron los 10 contratistas con mayor cantidad de pliegos ganados. Tal como puede verse en la Tabla 14, todos salvo un contratista (Firma 9) pertenecen al grupo con mayor participación.

Tabla 14: Primeras 10 empresas con mayor cantidad de pliegos ganados

\begin{tabular}{lrrr}
\hline & \multicolumn{3}{c}{ Pliegos } \\
\cline { 2 - 4 } Firmas & Presentados & Ganados & $\%$ Ganado \\
\hline Firma 1 & 720 & 264 & $37 \%$ \\
Firma 2 & 465 & 177 & $38 \%$ \\
Firma 3 & 301 & 137 & $46 \%$ \\
Firma 4 & 304 & 76 & $25 \%$ \\
Firma 5 & 259 & 69 & $27 \%$ \\
Firma 6 & 358 & 62 & $17 \%$ \\
Firma 7 & 233 & 60 & $26 \%$ \\
Firma 8 & 382 & 54 & $14 \%$ \\
Firma 9 & 90 & 54 & $60 \%$ \\
Firma 10 & 247 & 52 & $21 \%$ \\
\hline
\end{tabular}

Fuente: Elaboración propia en base a licitaciones disponibles online.

El objetivo de esta sección consiste en analizar si este grupo de firmas con mayor participación y mayor cantidad de pliegos ganados realizan bid skewing. Para poder determinar la heterogeneidad de la información entre las firmas se realiza un análisis de regresión y se comparan las firmas bajo los dos escenarios analizados con anterioridad: i) Contratistas vs Ingeniero y ii) Contratistas vs Consultor. Se realizan dos regresiones utilizando como variables dependientes el diferencial de ingresos resultante de utilizar diferentes vectores de precios (ecuaciones (7) y (8)). Como variables explicativas, se incluyeron distintas dummies referentes al ranking final en la licitación, las 10 primeras empresas con mayor cantidad de contratos ganados, y si las mismas ganaron la licitación. En línea con los resultados obtenidos en los primeros dos contraste de hipótesis, los coeficientes del ranking son positivos, decrecientes y estadísticamente significativos. La asimetría de información se refleja en la heterogeneidad de los efectos fijos por firma. En un extremo se encuentran las firmas 7 y 10 que son las que mayor bid skewing realizan. Por ejemplo, en el primer escenario la firma 7 obtiene ingresos $2.43 \%$ mayores a los que obtendría utilizando los precios re-escalados del ingeniero y un $1.87 \%$ aún más si esta firma es ganadora. En el otro extremo se encuentra la firma 3 que menor bid skewing realiza, donde sus ingresos son $0.97 \%$ menores a los que obtendría utilizando los precios re-escalados del ingeniero y un $0.33 \%$ aún menor si gana la licitación. En conclusión, existen contratistas con mayor información que otros. Los contratistas 7 y 10 desbalancean sus pliegos de manera más agresiva y son los únicos que parecieran tener información más precisa que el consultor. 
Tabla 15: Desbalanceo por firmas y ranking

\begin{tabular}{|c|c|c|c|c|}
\hline & \multicolumn{2}{|c|}{ Constratistas vs Ingeniero } & \multicolumn{2}{|c|}{ Constratistas vs Consultor } \\
\hline & coef & e.e. & coef & e.e \\
\hline Rank 1 & $0.0111^{* * *}$ & 0.0010 & $0.0128 * * *$ & 0.0013 \\
\hline Rank 2 & $0.0083^{* * *}$ & 0.0009 & $0.0089 * * *$ & 0.0011 \\
\hline Rank 3 & $0.0064^{* * *}$ & 0.0009 & $0.0071 * * *$ & 0.0012 \\
\hline Firma 1 & 0.0000 & 0.0021 & 0.0006 & 0.0027 \\
\hline Firma 2 & $-0.0046^{*}$ & 0.0026 & -0.0038 & 0.0034 \\
\hline Firma 3 & $-0.0097^{* * *}$ & 0.0034 & -0.0050 & 0.0044 \\
\hline Firma 4 & -0.0010 & 0.0029 & -0.0022 & 0.0037 \\
\hline Firma 5 & $0.0080^{* *}$ & 0.0032 & 0.0013 & 0.0041 \\
\hline Firma 6 & -0.0001 & 0.0026 & -0.0069 & 0.0033 \\
\hline Firma 7 & $0.0243^{* * *}$ & 0.0033 & $0.0228^{* * *}$ & 0.0043 \\
\hline Firma 8 & 0.0017 & 0.0024 & 0.0008 & 0.0031 \\
\hline Firma 9 & -0.0070 & 0.0073 & -0.0013 & 0.0093 \\
\hline Firma 10 & $0.0120^{* * *}$ & 0.0031 & $0.0147 * * *$ & 0.0040 \\
\hline Firma 1 \& Rank 1 & $-0.0091 * * *$ & 0.0035 & -0.0052 & 0.0045 \\
\hline Firma 2 \& Rank 1 & -0.0052 & 0.0043 & -0.0039 & 0.0055 \\
\hline Firma 3 \& Rank 1 & -0.0033 & 0.0052 & -0.0022 & 0.0066 \\
\hline Firma 4 \& Rank 1 & 0.0042 & 0.0059 & -0.0037 & 0.0075 \\
\hline Firma 5 \& Rank 1 & -0.0070 & 0.0062 & -0.0092 & 0.0080 \\
\hline Firma 6 \& Rank 1 & -0.0013 & 0.0062 & -0.0126 & 0.0079 \\
\hline Firma 7 \& Rank 1 & $0.0187^{* * *}$ & 0.0066 & 0.0068 & 0.0085 \\
\hline Firma 8 \& Rank 1 & 0.0039 & 0.0065 & -0.0073 & 0.0083 \\
\hline Firma 9 \& Rank 1 & 0.0023 & 0.0095 & $-0.0530^{* * *}$ & 0.0121 \\
\hline Firma 10 \& Rank 1 & $0.0251^{* * *}$ & 0.0069 & $0.0320^{* * *}$ & 0.0088 \\
\hline Constate & $0.0051 * * *$ & 0.0004 & $-0.0106^{* * *}$ & 0.0005 \\
\hline$R^{2}$ & 0.0197 & & 0.0137 & \\
\hline$N^{O}$ de Obs. & 21360 & & 21360 & \\
\hline
\end{tabular}

Fuente: Elaboración propia en base a licitaciones disponibles online.

Nota: $\mathrm{p}$-values ${ }^{*} \mathrm{p}<0.1, * * \mathrm{p}<0.05, * * * \mathrm{p}<0.01$

\section{Modelo - Contratistas aversos al riesgo}

Hasta aquí se trabajó con un modelo donde los contratistas son neutrales al riesgo. Bajo este escenario, los contratistas eligen sus precios unitarios para maximizar sus ingresos esperados sin considerar sus costos. La irrelevancia de los costos se debe a que los precios unitarios no afectan los costos esperados $^{9}$. En consecuencia, el ingreso de los contratistas sólo va a depender de los precios ofertados y de las cantidades finales. En este caso, los incentivos a desbalancear el pliego debido a la estrategia de quantity error explotation no tienen atenuantes.

En esta sección se presenta de manera teórica una variante del modelo que describe cómo se modificaría el análisis si los contratistas son aversos al riesgo. La estimación del modelo se encuentra fuera del alcance de este trabajo. La estrategia de quantity error explotation supone elegir precios unitarios por debajo de los costos para algunos ítems y por encima para otros. Si existe

\footnotetext{
${ }^{9}$ Los costos son irrelevantes en el segundo problema que tiene que resolver el contratista: cómo asignar los precios unitarios a cada uno de los componentes del proyecto.
} 
incertidumbre acerca de las cantidades finales, esta estrategia conlleva riesgos ya que para ciertas realizaciones de las cantidades el contratista podría obtener beneficios negativos. Un contratista averso al riesgo intentará reducir su exposición a este riesgo limitando cuánto desbalancea su pliego. Una forma de lograrlo es alineando los precios unitarios con los costos, por ejemplo, imponiendo el mismo mark-up para cada ítem y asegurarse beneficios positivos para cualquier realización de las cantidades. Mejor aún, el contratista debería imponer un mark-up cercano a cero para los ítems con mayor varianza y un mark-up alto para los ítems de menor varianza. En conclusión, un contratista averso al riesgo intentará no desbalancear excesivamente su pliego y alinear sus precios unitarios con sus costos unitarios. Por lo tanto, los incentivos a desbalancear el pliego debido a la estrategia de quantity error explotation son contrarrestados por el riesgo asociado a esta práctica.

En el modelo con aversión al riesgo, la heterogeneidad de los costos entre los contratistas y el grado de aversión al riesgo pueden juegar un rol importante. El desafío para el econometrista consiste en estimar los costos que se enfrentan los contratistas al momento de elegir los precios a ofertar, su grado de aversión al riesgo y sus expectativas respecto a los ajustes de las cantidades futuras, los cuales son desconocidos.

Al igual que en la sección 4.4 (contratistas vs. consultor), la hipótesis a contrastar es si, en promedio, los contratistas tienen mayor información que la contenida en la distribución empírica de los ajustes de cantidades al momento de presentar sus ofertas, pero ahora se permite que los contratistas tengan aversión al riesgo. Esta hipótesis se podría contrastar en el marco de un modelo que permite identificar separadamente todos estos parámetros desconocidos por el econometrista. A continuación se presenta una propuesta teórica del modelo con aversión al riesgo.

\subsection{Problema del Contratista}

El problema del contratista es maximizar el valor esperado de la utilidad de sus beneficios sujeto a un pliego total determinado:

$$
\begin{aligned}
& \operatorname{Max}_{\bar{B},\{b\}} E\left[U\left((b-c)^{\prime} X\right)\right] P(\text { Ganar } \mid \bar{B}) \\
& \text { sujeto a: } \\
& b^{\prime} Q=\bar{B}
\end{aligned}
$$

donde $c_{j}$ es el costo unitario de la tarea $j$ que es observado por el contratista, $X_{j}=Q_{j}\left(1+\Delta q_{j}\right)$ es la cantidad realizada de la tarea $j$ que es una variable aleatoria y $U($.) es una función de utilidad exponencial con la propiedad que el grado de aversión al riesgo es constante: $U(m)=1-e^{-\rho m}, \rho \geq 0$ donde $\rho$ es el coeficiente de aversión absoluta al riesgo ${ }^{10}$. Esta forma funcional surge de la necesidad de representar las preferencias del contratista independientemente de su nivel de riqueza inicial que no es observado.

Con aversión al riesgo, la elección óptima de $\bar{B}$ y $b$ dependen de los costos unitarios $c$. Al igual que en la sección 4, este trabajo sólo se focaliza en el problema de la elección óptima de los precios

\footnotetext{
${ }^{10}$ Para más detalle acerca de ésta medida de aversión absoluta al riesgo, ver Mass-Collel y otros (2004).
} 
dado un nivel $\bar{B}$ determinado ${ }^{11}$. Formalmente:

$$
\begin{aligned}
& V\left(c, \bar{B}, h_{X}(x)\right)=\operatorname{Max}_{\{b\}} E\left[U\left((b-c)^{\prime} X\right)\right] \\
& \text { sujeto } a: \\
& b^{\prime} Q=\bar{B}
\end{aligned}
$$

donde $V\left(c, \bar{B}, h_{X}(x)\right)$ es el máximo beneficio esperado que el contratista puede generar con un monto total $\bar{B}$, costos $c$ y una distribución de ajustes de cantidades $h_{X}(x)$. El econometrista no conoce cuál es la función de distribución de los ajustes de cantidades que describe las expectativas del contratista al momento de elegir sus precios a ofertar óptimos. Sin embargo, tiene información acerca de: i) la distribución empírica de los ajustes de cantidades (calculada en la sección 4.4), y ii) las cantidades que efectivamente se utilizaron. Si los contratistas pudieran predecir perfectamente las realizaciones, sus expectativas serían determinísticas y pueden ser representadas mediante una distribución Dirac igual a $\delta_{X=x_{a}}(x)^{12}$. La misma se encuentra centrada en $X=x_{a}$ y acumula toda la masa de probabilidad en $X=x_{a}$. Por lo tanto, se va a suponer que la función de distribución del contratista $h_{X}(x)$ va a estar comprendida entre las dos distribuciones mencionadas en i) y ii) de acuerdo a la siguiente relación:

$$
h_{X}(x)=\frac{f_{X}(x)+\gamma \delta_{X=x_{a}}(x)}{1+\gamma}
$$

donde el coeficiente $\gamma$ mide el grado de información que tiene el contratista respecto al consultor, $f_{X}(x)$ es la función de densidad asociada a la distribución empírica de los ajustes de cantidades y $\delta_{X=x_{a}}(x)$ es la distribución Dirac asociada a la realización observada de las cantidades. Si $\gamma=0$ no hay información privada, es decir, el contratista tiene la misma información que el consultor (tiene la misma información que la contenida en la distribución empírica de los ajustes de cantidades). Si $\gamma \rightarrow \infty$ el contratista tiene información privada.

Entonces, el vector de precios a ofertar óptimos $\left\{b^{*}\right\}$ va a ser aquel que cumpla la condición de primer orden de (10):

$$
\rho \int Q_{j} x_{j} e^{-\rho(b-c)^{\prime} Q x} \frac{f_{X}(x)+\gamma \delta_{X=x_{a}}(x)}{1+\gamma} d x=\tilde{\lambda} Q_{j}
$$

donde $x=\left(1+\Delta \tilde{q}^{e}\right), Q$ una matriz diagonal con las cantidades estimadas por el ingeniero y $\tilde{\lambda}$ es el multiplicador de Lagrange asociado a la restricción. Dividiendo ambos miembros por $Q_{j} \mathrm{y}$ operando algebraicamente, resulta:

$$
\int x_{j} e^{-m^{\prime} x} f(x) d x+\gamma e^{-m^{\prime} x} x_{a}=\lambda
$$

donde $m=\rho(b-c) Q$ es un vector que representa el mark-up del contratista y $\lambda=\frac{\widetilde{\lambda}}{\rho}(1+\gamma)$ es el multiplicador de Lagrange ajustado.

\footnotetext{
${ }^{11}$ Para la elección del monto a ofertar se resuelve: $\operatorname{Max}_{\{\bar{B}\}} V\left(c, \bar{B}, h_{X}(x)\right) P(\operatorname{Ganar} \mid \bar{B})$.

${ }^{12}$ Para más detalle acerca de esta distribución, ver Dirac, P. (1958).
} 


\subsection{Problema del Econometrista}

El problema del econometrista consiste en estimar los costos $(c)$, el coeficiente de aversión al riesgo $(\rho)$ y el coeficiente del nivel información $(\gamma)$ que racionalizan los precios ofertados $(b)$ satisfaciendo las condiciones de primer orden.

Se supone que el vector de costos unitarios se distribuye de acuerdo a $F_{c}$ con media $\bar{c}$ y que la realización de los costos $c$ es independiente a las cantidades estimadas por el ingeniero. Al momento de elegir su vector de precios $b$, el contratista observa una realización de sus costos unitarios $c$. Esta realización no es observada por el econometrista. Sin embargo, el econometrista sí observa los precios ofertados, las cantidades estimadas por el ingeniero y puede estimar la función de distribución de los ajustes de cantidades. Con esta información, es posible calcular el mark-up óptimo $m^{*}$ a partir de la condición de primer orden (11) para un $\gamma$ y $\lambda$ fijo. Despejando los precios de la ecuación del mark-up y re-escribiendo los costos como los costos medios más un error: $c_{j a}=\bar{c}_{j}+\varepsilon_{j a}$. Resulta:

$$
b_{j a} Q_{j a}=\frac{m_{j a}^{*}\left(\lambda_{a}, \gamma\right)}{\rho}+\bar{c}_{j} Q_{j a}+\varepsilon_{j a} Q_{j a}
$$

donde $a=1, \ldots, A$ indexa las licitaciones que participó el contratista y $j=1, \ldots, J$ los ítems o tareas comprendidos en cada licitación. Los costos y el coeficiente de aversión al riesgo pueden estimarse regresando (12) mediante mínimos cuadrados no lineales.

Para finalizar, el objetivo del econometrista es encontrar la combinación de $\rho, \gamma, \lambda$ y $c_{j a}$ que minimizan la variación no explicada de los costos. Formalmente, se construye el siguiente estimador:

$$
\{\widehat{\rho}, \widehat{\gamma}\}=\underset{\left\{m_{j a}^{*}\right\},\left\{\lambda_{a}\right\},\left\{\bar{c}_{j}\right\}, \rho, \gamma}{\operatorname{argmin}} \sum_{j} \sum_{a}\left(B_{j a}-\frac{m_{j a}^{*}\left(\lambda_{a}, \gamma\right)}{\rho}-\bar{c}_{j} Q_{j a}\right)^{2}
$$

En la sección A.2 del Anexo se presenta una propuesta metodológica de cómo comenzar a resolver computacionalmente el problema de econometrista. Por último, a continuación se analiza la identificación de los parámetros del modelo y se los verifica con una estimación de Monte Carlo.

\subsection{Identificación del Modelo}

El coeficiente de información $(\gamma)$ está identificado por la frecuencia con la que el contratista realiza bid skewing en la dirección correcta dada la realización real de las variaciones de las cantidades. La identificación de los costos $(c)$ depende de las cantidades nominales $(Q)$. Cuando la cantidad es grande, el bid skewing en ese ítem expone al contratista a mucho riesgo, y en consecuencia, el mismo va a ofertar un precio cercano a su costo. Y por último, la identificación del coeficiente de aversión al riesgo $(\rho)$ está medido por la magnitud del bid skewing cuando la cantidad nominal $(Q)$ es baja y el ítem en promedio tiende a ser sub o sobre-estimado (es decir que existen incentivos a desbalancear).

A continuación se realiza un ejercicio de Monte Carlo para verificar que las estimaciones de los parámetros sean cercanas a su valor real para un modelo más simple y con datos de licitaciones ficticios. El ejercicio de Monte Carlo que consiste en: i) fijar un conjunto de parámetros $(\rho, \gamma, \bar{c})$, 
ii) generar las muestras aleatorias sobre las distribuciones de $f_{X}(x)$ y $\delta_{X=x_{a}}(x)$, iii) computar el óptimo del contratista para cada muestra, iv) aplicar el estimador para cada muestra aleatoria, y v) comparar las estimaciones con el valor real de los parámetros.

Se considera un contratista que se presenta en 50 licitaciones que tienen como máximo 7 ítems. Las cantidades estimadas por el ingeniero $(Q)$, los costos $(c)$ y los costos medios $(\bar{c})$ fueron generados aleatoriamente. La distribución de $f_{X}(x)$ está construida con variables aleatorias que son independientes y están idénticamente distribuidas. Los resultados se encuentran en la Tabla 16, donde se puede ver que las estimaciones de los parámetros son muy cercanas a su valor real. De esta manera, también queda demostrado que el optimizador tiene un valor único.

Tabla 16: Simulación de Monte Carlo

\begin{tabular}{crccccc}
\hline \multirow{2}{*}{ Percentil* } & \multirow{2}{*}{ Valor Verdadero } & \multicolumn{5}{c}{ Valor Estimado (Percentiles) } \\
\cline { 3 - 7 } & & 5 & 25 & 50 & 75 & 95 \\
\hline$\rho$ & 0.25 & 0.2460 & 0.2482 & 0.2497 & 0.2515 & 0.2541 \\
$\gamma$ & 0.10 & 0.0961 & 0.0985 & 0.1000 & 0.1011 & 0.1030 \\
$\bar{c}_{1}$ & 0.3071 & 0.2694 & 0.2920 & 0.3077 & 0.3207 & 0.3422 \\
$\bar{c}_{2}$ & 0.7488 & 0.7185 & 0.7318 & 0.7480 & 0.7602 & 0.7775 \\
$\bar{c}_{3}$ & 0.1296 & 0.0864 & 0.1116 & 0.1321 & 0.1522 & 0.1762 \\
$\bar{c}_{4}$ & 0.7478 & 0.7120 & 0.7308 & 0.7467 & 0.7602 & 0.7781 \\
$\bar{c}_{5}$ & 0.8034 & 0.7672 & 0.7898 & 0.8074 & 0.8240 & 0.8432 \\
$\bar{c}_{6}$ & 0.3067 & 0.2714 & 0.2905 & 0.3063 & 0.3221 & 0.3392 \\
$\bar{c}_{7}$ & 0.2269 & 0.1732 & 0.1967 & 0.2237 & 0.2450 & 0.2854 \\
\hline
\end{tabular}

Nota: Presenta los percentiles de la distribucin de 100 muestras aleatorias.

Para la estimación de los parámetros utilizando los datos reales, hay que tener en cuenta que las distribuciones de $X$ exhibien correlaciones y masa de probabilidad. Por ejemplo, dos ítems pueden estar correlacionados y uno de los mismos posee masa de probabilidad en un evento en el cual no hay ajustes $\left(X_{j}=Q_{j}\right)$. En el Anexo se presenta una propuesta metodológica de cómo estimar la función de densidad $f_{X}(x)$ cuando los datos presentan estas características.

Una vez estimados estos parámetros, el análisis empírico a realizar consiste en:

1. Coeficiente de información $(\gamma)$ : Testear la hipótesis nula. Si $\gamma=0$, entonces los contratistas y consultor poseen la misma información acerca de la distribución empírica de las cantidades.

2. Vector de costos $(c)$ : Se pueden determinar cuáles fueron los componentes que tuvieron mayor o menor bid skewing a partir de la comparación entre los precios y los costos unitarios $(b-c)$.

3. Coeficiente de aversión al riesgo $(\rho)$ : Comparación entre contratistas.

4. Verificar si los resultados cambian con aversión al riesgo. Por ejemplo, con neutralidad al riesgo, la Firma 1 realiza poco bid skewing y la Firma 7 es la más agresiva. 


\section{Conclusión}

Este trabajo documenta empíricamente la existencia de bid skewing en los contratos de construcción del departamento de transporte de Michigan para el período 2001-2013.

Se desarrolla un modelo que permite distinguir y contrastar empíricamente las estrategias de front-end loading y quantity error exploitation por separado y supone que los contratistas son neutrales al riesgo. Para la estrategia de quantity error exploitation, el modelo considera que los contratistas obtienen su vector de precios óptimos dado unas creencias o información acerca de los ajustes de cantidades. A pesar de que no se observa la información que tienen los contratistas al momento de realizar sus ofertas, se infiere la calidad de la misma comparando los ingresos que obtienen en los datos con los ingresos que obtendrían si ofertaran precios unitarios distintos. Es decir, se compara los retornos que los contratistas efectivamente obtienen con los retornos que obtendrían utilizando las acciones óptimas bajo distintos supuestos informativos acerca de los ajustes de las cantidades.

Se encuentra evidencia estadísticamente significativa de que los contratistas están realizando bid skewing y el mismo viene explicado por la práctica de quantity error exploitation. Los contratistas obtienen, en promedio, retornos significativamente mayores de los que obtendrían si simplemente re-escalan los precios del ingeniero. Este resultado indica que existen asimetrías de la información entre los contratistas y el licitador.

Sin embargo, los contratistas no obtienen retornos significativamente mayores de los que obtendrían si conocieran la distribución empírica de los ajustes de cantidades. Esto indicaría cierto beneficio para el ingeniero del proyecto de utilizar información agregada de los ítems con mayor frecuencia al momento de calcular los precios y cantidades estimadas del proyecto.

Además, se encuentra evidencia de que existen asimetrías de la información entre los contratistas. El grupo ganador y con mayor participación en el mercado de licitaciones es el que mayor bid skewing realiza. En particular, la firmas 7 y 10 poseen información más precisa acerca de la variación futura de las cantindades y actúan sobre ella desbalanceando sus pliegos de manera más agresiva.

Por último, se desarrolla de manera teórica un segundo modelo que considera que los contratistas son aversos al riesgo y tienen heterogeneidad en los costos. En este modelo, el contratista averso al riesgo intentará no desbalancear excesivamente su pliego y alineará sus precios unitarios con sus costos unitarios. Por lo tanto, los incentivos a desbalancear el pliego debido a la estrategia de quantity error explotation son contrarrestados por el riesgo asociado a esta práctica. 


\section{Bibliografía}

ARYAL, G. y GABRIELLI, M. F. (2013): "Testing for Collusion in Asymmetric First Price Auction", International Journal of Industrial Organization, 31, 26-35.

ATHEY, S. y LEVIN, J. (2001), "Information and Competition in U.S. Forest Service Timber Auctions", Journal of Political Economy, 109(2), 375-417.

ATHEY, S., COEY, D. y LEVIN, J. (2011): "Set-asides and Subsidies in Auctions", Discussion paper, National Bureau of Economic Research.

BAJARI, P., HOUGHTON, S. y TADELIS, S. (2011): "Bidding for Incomplete Contracts: An Empirical Analysis of Adaptation Costs", University of Minnesota, Texas A\&M University and UC Berkeley.

BAJARI, P. y YE, L. (2003): "Deciding Between Competition and Collusion", The Review of Economics and Statistics, 85(4), 971-989.

CATTELL, D.W. (1984): "A model for item price loading by building contractors", Unpublished paper, Department of Quantity Surveying, University of the Witwatersrand, Johannesburg.

CATTELL, D.W. (1987): "Item price loading", PACE'87 Progress in Architecture, Construction and Engineering proceedings, Johannesburg, July, 2(3),Vol.II, Session 3, pp.1-20.

CATTELL, D.W., BOWEN, P.A. y KAKA, A.P. (2008): "A simplified unbalanced bidding model", Construction Management and Economics, 26(12), 1283-1290.

CATTELL, D.W., BOWEN, P.A. y KAKA, A.P. (2010): "The risks of unbalanced bidding", Construction Management and Economics, 28(4), 333-334.

DIEKMANN, J.E., MAYER, R.H. Jr. y STARK, R.M. (1982): "Coping with uncertainty in unit price contracting", American Society of Civil Engineers, Journal of the Construction Division, 108(C03), 379-389.

DIRAC, P. (1958): "Principles of quantum mechanics", Oxford at the Clarendon Press, 4th Edition, pp.60-63.

FLAMBARD, V. y PERRIGNE, I. (2006): "Asymmetry in Procurement Auctions: Evidence from Snow Removal Contracts", The Economic Journal, 116(514), 1014-1036.

GATES, M. (1959): "Aspects of competitive bidding", Annual Report, Connecticut Society of Civil Engineers.

GATES, M. (1967): "Bidding strategies and probabilities", Journal of the Construction Division, American Society of Civil Engineers, vol. 93, n. C01, Proc paper 5159, Mar., pp. 75-107.

GUERRE, E., PERRIGNE, I. y VUONG, Q. (2000): "Optimal nonparametric estimation of first- 
price auctions", Econometrica, 68:525-574.

GUERRE, E., PERRIGNE, I. y VUONG, Q. (2009): "Nonparametric identification of risk aversion in first-price auctions under exclusion restrictions", Econometrica, 77 (4):1193-1227.

HAILE, P.A., HONG, H. y SHUM, M. (2004): "Nonparametric Tests for Common Values in FirstPrice Sealed-Bid Auctions", Yale U., Duke U. and Johns Hopkins U.

KAWAI, K. (2011): "Auction Design and the Incentives to Invest: Evidence from Procurement Auctions", NYU Stern.

KRASNOKUTSKAYA, E. (2011): "Identication and estimation of auction models with unobserved heterogeneity", The Review of Economic Studies, 78(1), 293.

KRASNOKUTSKAYA, E. y SEIM, K. (2008): "Bid preference programs and participation in highway procurement auctions", University of Pennsylvania, Wharton.

LAFFONT, J. y VUONG, Q. (1996): "Structural analysis of auction data", American Economic Review, 86:414-420.

MAS-COLELL, A., WHINSTON M.D., and GREEN J.R. (1995), "Microeconomic Theory", Oxford University Press,pp. 183-194.

PAARSCH, H. y HONG, H. (2006): "An Introduction to the Structural Econometrics of Auction Data", MIT Press.

PERRIGNE, I. y VUONG, Q. (1999): "Structural econometrics of first-price auctions: A survey of methods", Canadian Journal of Agricultural Economics, 47:203223.

PERRIGNE, I. y VUONG, Q. (2008): "Auctions: Empirics", S. Durlauf and L. Blume, Palgrave McMillan, second edition.

PORTER, R.H., y ZONA, J.D. (1993): "Detection of Bid Rigging in Procurement Auctions", Journal of Political Economy, 101(3), 518-38.

PORTER, R.H., y ZONA, J.D. (1999): "Ohio School Milk Markets: An Analysis of Bidding", The RAND Journal of Economics, 30(2), 263-288.

SKLAR, A. (1959): "Fonctions de répartition à n dimensions et leurs marges", Publ. Inst. Statist. Univ. Paris 8: 229-231.

STARK, R.M. (1968): "Unbalanced bidding models - theory", American Society of Civil Engineers, Journal of the Construction Division, 94(C02), 197-209.

STARK, R.M. (1972): "Unbalancing of tenders", Proceedings of the Institute of Civil Engineers, Technical Note 59, vol. 51, pp. 391-392.

STARK, R.M. (1974): "Unbalanced highway contract tendering”. Operational Research Quarterly, 
25(3), 373-388.

TEICHOLZ, P.M. y ASHLEY, D.B. (1978): "Optimal bid prices for unit price contract", American Society of Civil Engineers, Journal of the Construction Division, 104(1), 57-67.

\section{A. Anexo}

\section{A.1. Tablas Adicionales}

Tabla A1: Estadísticas descriptivas de los pliegos según participación

\begin{tabular}{|c|c|c|c|c|c|c|}
\hline & Participación & Obs. & Media & Des. Est & Min. & Max. \\
\hline Pliego Ganador (\$) & {$[1-10]$} & 175 & 727,868 & $1,218,253$ & 13,470 & $7,553,670$ \\
\hline Markup: (Pliego Ganador-Estimado)/Estimado & {$[1-10]$} & 175 & -0.1235 & 0.1669 & -0.7488 & 0.2341 \\
\hline Ex Post Markup: (Ingreso-Estimado)/Estimado & {$[1-10]$} & 175 & -0.1331 & 0.1773 & -0.6938 & 0.4357 \\
\hline Contratistas por contrato & {$[1-10]$} & 175 & 6.35 & 3.65 & 1 & 21 \\
\hline Pliego Ganador $(\$)$ & {$[11-50]$} & 595 & 591,891 & $1,034,408$ & 10,700 & $14,900,000$ \\
\hline Markup: (Pliego Ganador-Estimado)/Estimado & {$[11-50]$} & 595 & -0.0829 & 0.1559 & -0.7209 & 1.0822 \\
\hline Ex Post Markup: (Ingreso-Estimado)/Estimado & {$[11-50]$} & 595 & -0.1053 & 0.1675 & -0.9204 & 0.7463 \\
\hline Contratistas por contrato & {$[11-50]$} & 595 & 6.15 & 3.81 & 1 & 19 \\
\hline Pliego Ganador (\$) & {$[51-100]$} & 688 & 774,208 & $1,038,026$ & 8,069 & $8,866,790$ \\
\hline Markup: (Pliego Ganador-Estimado)/Estimado & {$[51-100]$} & 688 & -0.0889 & 0.1403 & -0.5423 & 0.5816 \\
\hline Ex Post Markup: (Ingreso-Estimado)/Estimado & {$[51-100]$} & 688 & -0.1104 & 0.1567 & -0.8225 & 0.9490 \\
\hline Contratistas por contrato & {$[51-100]$} & 688 & 5.47 & 3.22 & 1 & 21 \\
\hline Pliego Ganador (\$) & {$[+100]$} & 1933 & 966,515 & $1,544,042$ & 9,711 & $40,500,000$ \\
\hline Markup: (Pliego Ganador-Estimado)/Estimado & {$[+100]$} & 1933 & -0.0618 & 0.1510 & -0.7295 & 1.1569 \\
\hline Ex Post Markup: (Ingreso-Estimado)/Estimado & {$[+100]$} & 1933 & -0.0867 & 0.1794 & -0.9365 & 2.0490 \\
\hline Contratistas por contrato & {$[+100]$} & 1933 & 4.88 & 3.01 & 1 & 23 \\
\hline
\end{tabular}

Fuente: Elaboración propia en base a licitaciones disponibles online.

Tabla A2: Evidencia de Quantity Error Exploitation (nivel del ítem)

\begin{tabular}{|c|c|c|c|c|c|c|c|c|c|c|}
\hline \multirow[t]{2}{*}{ Rank } & \multirow[t]{2}{*}{ Obs. } & \multirow[t]{2}{*}{ Media } & \multirow[t]{2}{*}{ Des. Est. } & \multirow[t]{2}{*}{ T-stat } & \multicolumn{5}{|c|}{ Percentiles } & \multirow[t]{2}{*}{$\operatorname{Prob}\left(R_{q e}\right)>0$} \\
\hline & & & & & 5 & 25 & 50 & 75 & 95 & \\
\hline 1 & 192663 & 0.00028 & 0.01616 & $7.6^{* * *}$ & -0.0115 & -0.0007 & 0.0000 & 0.0006 & 0.0129 & $43.0 \%$ \\
\hline 2 & 190411 & 0.00023 & 0.01615 & $6.3^{* * *}$ & -0.0115 & -0.0007 & 0.0000 & 0.0005 & 0.0126 & $41.2 \%$ \\
\hline 3 & 169591 & 0.00020 & 0.01573 & $5.2^{* * *}$ & -0.0115 & -0.0008 & 0.0000 & 0.0005 & 0.0125 & $39.8 \%$ \\
\hline
\end{tabular}

Fuente: Elaboración propia en base a licitaciones disponibles online.

Nota: $\mathrm{p}$-values ${ }^{*} \mathrm{p}<0.1, * * \mathrm{p}<0.05, * * * \mathrm{p}<0.01$ 
Tabla A3: Evidencia de Quantity Error Exploitation según participación (nivel del ítem)

\begin{tabular}{|c|c|c|c|c|c|c|c|c|c|c|}
\hline \multirow[t]{2}{*}{ Participación ${ }^{*}$} & \multirow[t]{2}{*}{ Obs. } & \multirow[t]{2}{*}{ Media } & \multirow[t]{2}{*}{ Des. Est. } & \multirow[t]{2}{*}{ T-stat } & \multicolumn{5}{|c|}{ Percentiles } & \multirow[t]{2}{*}{$\operatorname{Prob}\left(R_{q e}\right)>0$} \\
\hline & & & & & 5 & 25 & 50 & 75 & 95 & \\
\hline$[1-10]$ & 8755 & 0.00017 & 0.02019 & 0.8 & -0.01415 & -0.00074 & 0.00000 & 0.00088 & 0.01622 & $45.2 \%$ \\
\hline$[11-50]$ & 28922 & 0.00020 & 0.01614 & $2.1^{* *}$ & -0.01370 & -0.00084 & 0.00000 & 0.00091 & 0.01565 & $44.5 \%$ \\
\hline$[51-100]$ & 37315 & 0.00026 & 0.01514 & $3.3^{* * *}$ & -0.01172 & -0.00070 & 0.00000 & 0.00073 & 0.01351 & $43.2 \%$ \\
\hline$[+100]$ & 117671 & 0.00032 & 0.01614 & $6.7^{* * *}$ & -0.01080 & -0.00059 & 0.00000 & 0.00053 & 0.01182 & $42.4 \%$ \\
\hline
\end{tabular}

Fuente: Elaboración propia en base a licitaciones disponibles online.

Nota: $\mathrm{p}$-values $* \mathrm{p}<0.1,{ }^{* *} \mathrm{p}<0.05, * * * \mathrm{p}<0.01$ 
Tabla A4: Primeros 25 Items con mayor frecuencia

\begin{tabular}{|c|c|c|c|c|c|c|c|}
\hline Categoría 1 & Categoría 2 & Item - Descripción & Unid. & Freq. & $1+\bar{q}_{j}^{e}$ & $p_{(10)}\left[\pi_{j}\right]$ & $p_{(90)}\left[\pi_{j}\right]$ \\
\hline Incidental Construction & Temporary Traffic Control for Construction Zone Operations & Lighted Arrow, Type C, Oper & $\mathrm{Ea}$ & 1876 & 0.88 & 0.00 & 2.52 \\
\hline Incidental Construction & Temporary Traffic Control for Construction Zone Operations & Lighted Arrow, Type C, Furn & $\mathrm{Ea}$ & 1873 & 0.88 & 0.16 & 1.85 \\
\hline Earthwork & Soil Erosion and Sedimentation Control & Erosion Control, Silt Fence & $\mathrm{Ft}$ & 1429 & 0.54 & 0.51 & 1.51 \\
\hline Incidental Construction & Temporary Traffic Control for Construction Zone Operations & Sign, Type B, Temp, Prismatic, Furn & Sft & 1351 & 0.82 & 0.31 & 1.69 \\
\hline Incidental Construction & Temporary Traffic Control for Construction Zone Operations & Sign, Type B, Temp, Prismatic, Oper & Sft & 1350 & 0.81 & 0.01 & 2.14 \\
\hline Incidental Construction & Temporary Traffic Control for Construction Zone Operations & Plastic Drum, High Intensity, Lighted, Oper & $\mathrm{Ea}$ & 1317 & 0.94 & 0.00 & 2.11 \\
\hline Incidental Construction & Temporary Traffic Control for Construction Zone Operations & Plastic Drum, High Intensity, Lighted, Furn & $\mathrm{Ea}$ & 1314 & 0.94 & 0.28 & 1.93 \\
\hline Incidental Construction & Temporary Traffic Control for Construction Zone Operations & Sign Cover & $\mathrm{Ea}$ & 1128 & 0.39 & 0.00 & 1.88 \\
\hline Incidental Construction & Permanent Pavement Markings & Pavt Mrkg, Waterborne, 4 inch, Yellow & Ft & 1121 & 0.98 & 0.41 & 3.05 \\
\hline Earthwork & Roadway Earthwork & Excavation, Earth & Cyd & 1090 & 0.95 & 0.52 & 2.30 \\
\hline Incidental Construction & Temporary Traffic Control for Construction Zone Operations & Sign, Type B, Temp, Furn & Sft & 1088 & 0.96 & 0.38 & 1.74 \\
\hline Incidental Construction & Temporary Traffic Control for Construction Zone Operations & Sign, Type B, Temp, Oper & Sft & 1087 & 0.95 & 0.01 & 1.56 \\
\hline Earthwork & Removing Miscellaneous Structures and Materials & Curb and Gutter, Rem & $\mathrm{Ft}$ & 1085 & 1.10 & 0.50 & 1.99 \\
\hline Incidental Construction & Temporary Traffic Control for Construction Zone Operations & Barricade, Type III, High Intensity, Lighted, Oper & $\mathrm{Ea}$ & 1021 & 0.85 & 0.00 & 2.05 \\
\hline Incidental Construction & Temporary Traffic Control for Construction Zone Operations & Barricade, Type III, High Intensity, Lighted, Furn & $\mathrm{Ea}$ & 1020 & 0.85 & 0.23 & 1.66 \\
\hline HMA Pavements \& Surface Treatments & HMA Construction Practices & HMA Approach & Ton & 1020 & 1.03 & 0.75 & 1.62 \\
\hline Incidental Construction & Permanet Pavement Markings & Pavt Mrkg, Waterborne, 4 inch, White & $\mathrm{Ft}$ & 1015 & 0.97 & 0.34 & 3.74 \\
\hline Earthwork & Removing Miscellaneous Structures and Materials & Pavt, Rem & Syd & 989 & 1.06 & 0.43 & 2.27 \\
\hline Drainage Features & Drainage Structures & Dr Structure Cover & $\mathrm{Ea}$ & 947 & 0.96 & 0.67 & 1.49 \\
\hline Earthwork & Removing Miscellaneous Structures and Materials & Sidewalk, Rem & Syd & 939 & 1.14 & 0.51 & 2.13 \\
\hline Incidental Construction & Temporary Traffic Control for Construction Zone Operations & Plastic Drum, High Intensity, Furn & $\mathrm{Ea}$ & 930 & 0.91 & 0.19 & 1.56 \\
\hline Incidental Construction & Temporary Traffic Control for Construction Zone Operations & Plastic Drum, High Intensity, Oper & $\mathrm{Ea}$ & 928 & 0.91 & 0.00 & 1.15 \\
\hline HMA Pavements \& Surface Treatments & HMA Construction Practices & Cold Milling HMA Surface & Syd & 909 & 0.97 & 0.43 & 2.71 \\
\hline HMA Pavements \& Surface Treatments & HMA Construction Practices & Hand Patching & Ton & 895 & 0.67 & 0.55 & 2.11 \\
\hline Bases & Subbase & Subbase, CIP & Cyd & 810 & 0.81 & 0.62 & 1.79 \\
\hline
\end{tabular}

Fuente: Elaboración propia en base a licitaciones disponibles online. 


\section{A.2. Metodología de Estimación}

El problema se resuelve para un contratista determinado en el cual se tiene información acerca de todas las licitaciones que participó $(a=1, \ldots, A)$ y de los ítems o tareas comprendidos en cada licitación $(j=1, \ldots, J)$. Las dimensiones de los parámetros a estimar es la siguiente: matriz de costos $\left(C_{J \times A}\right)$, vector de costos medios $\left(\bar{c}_{J \times 1}\right)$, coeficiente de aversión al riesgo $\left(\rho_{1 \times 1}\right)$, coeficiente de nivel información $\left(\gamma_{1 \times 1}\right)$ y multiplicador de Lagrande $\left(\lambda_{A \times 1}\right)$.

Para obtener estos parámetros se deberá resolver un problema de optimización no lineal en el cual se minimiza la función objetivo (13) sujeto a que la condición de primer orden (12) se cumpla. Este problema se puede programar en MATLAB utilizando el solver KNITRO (que significa Nonlinear Interior point Trust Region Optimization). Este solver es un state of the art algorithm que resuelve problemas de optimización lineales, cuandráticos y no lineales ya sean convexos o no convexos.

Con aversión al riesgo, la interdependencia entre las variables aleatorias $X_{j}$ ahora es relevante. A continuación se presenta una propuesta de cómo estimar la función de densidad asociada a la distribución empírica de los ajustes de cantidades $f_{X}(x)$ y de cómo resolver analíticamente la integral de la condición de primer orden.

\section{A.2.1. Estimación de la función de densidad $f_{X}(x)$}

Para estimar la función de densidad $f_{X}$ se aplica el teorema de Sklar (1959) el cual establece que cualquier función de distribución multivariada conjunta puede ser expresada en términos de sus funciones de distribución marginales y la cópula, que describe la dependencia entre las variables.

\section{Función de distribución marginal}

Sea $F_{j}$ la función de distribución marginal acumulada de la variable aleatoria $X_{j}=Q_{j}\left(1+\Delta \tilde{q}_{j}\right)$, para $\Delta \tilde{q}_{j}$ que se distribuye:

$$
\Delta \tilde{q}_{j \sim} \sim \begin{cases}p_{1} & 0 \\ p_{2} & -1 \\ 1-p_{1}-p_{2} & \sim N\left(\mu, s^{2}\right)\end{cases}
$$

Esta distribución posee masa de probabilidad en 0 y en -1 que corresponden, respectivamente, a eventos en los que no hay ajustes o en los que el ítem es eliminado; estos eventos ocurren frecuentemente en los datos.

Para cada ítem $j$ se estima $p_{1 j}, p_{2 j}, \mu_{j}$ y $s_{j}^{2}$ y luego se estima la función de distribución marginal acumulada $\hat{F}_{j}$. Estas distribuciones marginales no son continuas. Por lo tanto, la cópula no esta no-paramétricamente identificada. Por esa razón, y para evitar la maldición de la dimensionalidad, se supone que la cópula tiene una forma Gaussiana cuya matriz de correlación tiene una estructura de factores. Para ello se define conceptualmente la variable aleatoria $U_{j}$ que se distribuye 
uniformemente entre 0 y 1 de forma que $X_{j}=F_{j}^{-1}\left(U_{j}\right)$, donde la función $F_{j}^{-1}$ está definida sin arbitrariedades porque $U_{j}$ tiene un soporte continuo.

\section{Cópula Gaussiana}

Sea $\Phi$ la función de distribución acumulada de una variable normal estandarizada y $\Phi^{-1} \mathrm{su}$ inversa, la función de quantiles. Se considera el siguiente vector aleatorio:

$$
\left(Z_{1}, Z_{2}, \ldots, Z_{J}\right)=\left(\Phi^{-1}\left(U_{1}\right), \Phi^{-1}\left(U_{2}\right), \ldots, \Phi^{-1}\left(U_{J}\right)\right)
$$

Por construcción, cada componente de este vector se distribuye normal estándar. El supuesto de cópula Gaussiana implica que estas variables se distribuyan conjuntamente normal (o que el vector sea una normal multivariada): $\vec{Z} \sim(0, \Sigma)$, donde $\Sigma$ es la matriz de correlación. Para reducir el número de parámetros se supone que esta matriz puede representarse como un modelo de factores:

$$
\Sigma=L L^{\prime}+D
$$

donde $L$ es la matriz de factor loadings de orden $J \times K$ para $K<J$ y $D$ es una matriz diagonal de orden $J$ que asegura que todos los elementos de la diagonal principal de $\Sigma$ sean igual a 1 . La distribución conjunta esta parametrizada por la matriz L. Para estimar estos parámetros se utiliza un estimador de máxima verosimilitud:

$$
\log V\left(L \mid X, p, \mu, s^{2}\right)=\sum_{a=1}^{A} \log V_{a}\left(L \mid X_{a}, p, \mu, s^{2}, F\right)
$$

donde $\log V_{a}\left(L \mid X_{a}, p, \mu, s^{2}, F\right)$ es la densidad/probabilidad de obtener el vector $X_{a}$ dados los parámetros $\left(L, p, \mu, s^{2}\right)$. La optimización es solamente sobre los parámetros en $L$, los otros parámetros son fijados en los valores estimados anteriormente.

\section{A.2.2. Resolución analítica de la integral $\int x_{j} e^{-x^{\prime} m} f(x) d x$}

El objetivo en esta sección consiste en estimar con mayor precisión la integral de la condición de primer orden. Para ello se tratará de resolverla analíticamente separando aquellos componentes que son aleatorios de los que no lo son.

Sean $v \sim N(0, I)$ el vector de factores de orden $K \times 1, e \sim N(0,1)$ el vector de ítems de orden $J \times 1, L$ la matriz de factor loadings de orden $J \times K$ y $\sigma_{j}=\left(D_{j j}\right)^{1 / 2}$ la varianza del ítem $j . \mathrm{El}$ elemento $z_{j}$ se lo puede escribir como $z_{j}=L_{j} v+\sigma_{j} e_{j}$ donde $z_{j} \sim N(0,1)$ porque $L_{j}^{\prime} L_{j}+\sigma_{j}^{2}=1$. Sea $x_{j}$ una transformación de $z_{j}$ tal que $x_{j}=\eta_{j}\left(z_{j}\right)=\eta_{j}\left(L_{j} v+\sigma_{j} e_{j}\right)$. Remplazando $x_{j}$ en la integral, resulta:

$$
\int x_{j} e^{-x^{\prime} m} f(x) d x=\int \eta_{j}\left(L_{j} v+\sigma_{j} e_{j}\right) e^{-\eta(L v+\sigma e)^{\prime} m} \phi\left(e_{1}\right) \ldots \phi\left(e_{J}\right) \phi\left(v_{1}\right) \ldots \phi\left(v_{F}\right) d e d v
$$

donde $\phi($.$) es una función de densidad normal estándar univariada. Reordenando los términos y$ agrupando convenientemente:

$$
=\int\left(\frac{\int \eta_{j}\left(L_{j} v+\sigma_{j} e_{j}\right) e^{-\eta_{j}\left(L_{j} v+\sigma_{j} e_{j}\right) m_{j}} \phi\left(e_{j}\right) d e_{j}}{\int e^{-\eta_{j}\left(L_{j} v+\sigma_{j} e_{j}\right)^{\prime} m_{j}} \phi\left(e_{j}\right) d e_{j}}\right) \prod_{k=1}^{J}\left(\int e^{-\eta_{k}\left(L_{k} v+\sigma_{k} e_{k}\right) m_{k}} \phi\left(e_{k}\right) d e_{k}\right) \phi\left(v_{1}\right) \ldots \phi\left(v_{F}\right) d v
$$


Realizando el cambio de variables $e_{k}=\frac{z_{k}-L_{k} v_{s}}{\sigma_{k}}$, ahora la integral sólo tiene un componente aleatorio dado por el vector $v$. Entonces, generando 10,000 muestras aleatorias de $v$, la integral puede ser descompuesta de la siguiente manera:

$$
=\frac{1}{S} \sum_{s=1}^{10,000} \frac{r_{j}\left(m, v_{s}\right)}{\tau_{j}\left(m, v_{s}\right)} \prod_{k=1}^{J=317} \tau_{k}\left(m, v_{s}\right)
$$

donde $\tau_{k}\left(m, v_{s}\right)$ y $r_{k}\left(m, v_{s}\right)$ se definen como:

$$
\begin{aligned}
\tau_{k}\left(m, v_{s}\right) & =\int e^{-\eta_{k}\left(z_{k}\right) m_{k}} \frac{1}{\sigma_{k}} \phi\left(\frac{z_{k}-L_{k} v_{s}}{\sigma_{k}}\right) d z_{k} \\
r_{k}\left(m, v_{s}\right) & =\int \eta_{k}\left(z_{k}\right) e^{-\eta_{k}\left(z_{k}\right) m_{k}} \frac{1}{\sigma_{k}} \phi\left(\frac{z_{k}-L_{k} v_{s}}{\sigma_{k}}\right) d z_{k}
\end{aligned}
$$

La función $\eta_{k}\left(z_{k}\right)$ se la puede escribir como:

$$
\begin{aligned}
z \in\left(-\infty, \underline{t}_{0 k}\right): \eta_{k}\left(z_{k}\right) & =\Phi^{-1}\left[\frac{\Phi\left(z_{k}\right)}{\left(1-p_{0 k}-p_{1 k}\right)}\right] s_{k}+\mu_{k} \\
z \in\left(\underline{t}_{0 k}, \bar{t}_{0 k}\right): \eta_{k}\left(z_{k}\right) & =0 \\
z \in\left(\bar{t}_{0 k}, \underline{t}_{1 k}\right): \eta_{k}\left(z_{z}\right) & =\Phi^{-1}\left[\frac{\Phi\left(z_{k}\right)-p_{0 k}}{\left(1-p_{0 k}-p_{1 k}\right)}\right] s_{k}+\mu_{k} \\
z \in\left(\underline{t}_{1 k}, \bar{t}_{1 k}\right): \eta_{k}(z) & =1 \\
z \in\left(\bar{t}_{1 k}, \infty\right): \eta_{k}(z) & =\Phi^{-1}\left[\frac{\Phi\left(z_{k}\right)-p_{0 k}-p_{1 k}}{\left(1-p_{0 k}-p_{1 k}\right)}\right] s_{k}+\mu_{k}
\end{aligned}
$$

donde los límites se obtienen de la siguiente manera:

$$
\begin{aligned}
& P\left(x_{k}<0\right)=\left(1-p_{0 k}-p_{1 k}\right) \Phi\left(-\frac{\mu_{k}}{s_{k}}\right) \Rightarrow \underline{t}_{0 k}=\Phi^{-1}\left[\left(1-p_{0 k}-p_{1 k}\right) \Phi\left(-\frac{\mu_{k}}{s_{k}}\right)\right] \\
& P\left(x_{k} \leq 0\right)=p_{0 k}+\left(1-p_{0 k}-p_{1 k}\right) \Phi\left(-\frac{\mu_{k}}{s_{k}}\right)=\Phi\left(\bar{t}_{0 k}\right) \Rightarrow \bar{t}_{0 k}=\Phi^{-1}\left[p_{0 k}+\left(1-p_{0 k}-p_{1 k}\right) \Phi\left(-\frac{\mu_{k}}{s_{k}}\right)\right] \\
& P\left(x_{k}<1\right)=p_{0 k}+\left(1-p_{0 k}-p_{1 k}\right) \Phi\left(\frac{1-\mu_{k}}{s_{k}}\right)=\Phi\left(\underline{t}_{1 k}\right) \Rightarrow \underline{t}_{1 k}=\Phi^{-1}\left[p_{0 k}+\left(1-p_{0 k}-p_{1 k}\right) \Phi\left(\frac{1-\mu_{k}}{s_{k}}\right)\right] \\
& P\left(x_{k} \leq 1\right)=p_{0 k}+p_{1 k}+\left(1-p_{0 k}-p_{1 k}\right) \Phi\left(\frac{1-\mu_{k}}{s_{k}}\right)=\Phi\left(\bar{t}_{1 k}\right) \Rightarrow \bar{t}_{1 k}=\Phi^{-1}\left[p_{0 k}+p_{1 k}+\left(1-p_{0 k}-p_{1 k}\right) \Phi\left(\frac{1-\mu_{k}}{s_{k}}\right)\right]
\end{aligned}
$$


Entonces, $\tau_{k}\left(m, v_{s}\right)$ y $r_{k}\left(m, v_{s}\right)$ quedan definidas como:

$$
\begin{aligned}
& \tau_{k}\left(m, v_{s}\right)=\int e^{-\eta_{k}\left(z_{k}\right) m_{k}} \frac{1}{\sigma_{k}} \phi\left(\frac{z_{k}-L_{k} v_{s}}{\sigma_{k}}\right) d z_{k} \\
& =\int_{-\infty}^{\underline{t}_{0 k}} e^{-\left(\Phi^{-1}\left[\frac{\Phi\left(z_{k}\right)}{\left(1-p_{0 k}-p_{1 k}\right)}\right] s_{k}+\mu_{k}\right) m_{k}} \frac{1}{\sigma_{k}} \phi\left(\frac{z_{k}-L_{k} v_{s}}{\sigma_{k}}\right) d z_{k} \\
& +\int_{\underline{t}_{0 k}}^{\bar{t}_{0 k}} \frac{1}{\sigma_{k}} \phi\left(\frac{z_{k}-L_{k} v_{s}}{\sigma_{k}}\right) d z_{k} \\
& +\int_{\bar{t}_{0 k}}^{\underline{t}_{1 k}} e^{-\left(\Phi^{-1}\left[\frac{\Phi\left(z_{k}\right)-p_{0 k}}{\left(1-p_{0 k}-p_{1 k}\right)}\right] s_{k}+\mu_{k}\right) m_{k}} \frac{1}{\sigma_{k}} \phi\left(\frac{z_{k}-L_{k} v_{s}}{\sigma_{k}}\right) d z_{k} \\
& +\int_{\underline{t}_{1 k}}^{\bar{t}_{1 k}} e^{-m} \frac{1}{\sigma_{k}} \phi\left(\frac{z_{k}-L_{k} v_{s}}{\sigma_{k}}\right) d z_{k} \\
& +\int_{\bar{t}_{1 k}}^{\infty} e^{-\left(\Phi^{-1}\left[\frac{\Phi\left(z_{k}\right)-p_{0 k}-p_{1 k}}{\left(1-p_{0 k}-p_{1 k}\right)}\right] s_{k}+\mu_{k}\right) m_{k}} \frac{1}{\sigma_{k}} \phi\left(\frac{z_{k}-L_{k} v_{s}}{\sigma_{k}}\right) d z_{k} \\
& r_{k}\left(m, v_{s}\right)=\int \eta_{k}\left(z_{k}\right) e^{-\eta_{k}\left(z_{k}\right) m_{k}} \frac{1}{\sigma_{k}} \phi\left(\frac{z_{k}-L_{k} v_{s}}{\sigma_{k}}\right) d z_{k} \\
& =\int_{-\infty}^{\underline{t}_{0 k}}\left(\Phi^{-1}\left[\frac{\Phi\left(z_{k}\right)}{\left(1-p_{0 k}-p_{1 k}\right)}\right] s_{k}+\mu_{k}\right) e^{-\left(\Phi^{-1}\left[\frac{\Phi\left(z_{k}\right)}{\left(1-p_{0 k}-p_{1 k}\right)}\right] s_{k}+\mu_{k}\right) m_{k}} \frac{1}{\sigma_{k}} \phi\left(\frac{z_{k}-L_{k} v_{s}}{\sigma_{k}}\right) d z_{k} \\
& +\int_{\underline{t}_{0 k}}^{\bar{t}_{0 k}} 0 \frac{1}{\sigma_{k}} \phi\left(\frac{z_{k}-L_{k} v_{s}}{\sigma_{k}}\right) d z_{k} \\
& +\int_{\bar{t}_{0 k}}^{\underline{t}_{1 k}}\left(\Phi^{-1}\left[\frac{\Phi\left(z_{k}\right)-p_{0 k}}{\left(1-p_{0 k}-p_{1 k}\right)}\right] s_{k}+\mu_{k}\right) e^{-\left(\Phi^{-1}\left[\frac{\Phi\left(z_{k}\right)-p_{0 k}}{\left(1-p_{0 k}-p_{1 k}\right)}\right] s_{k}+\mu_{k}\right) m_{k}} \frac{1}{\sigma_{k}} \phi\left(\frac{z_{k}-L_{k} v_{s}}{\sigma_{k}}\right) d z_{k} \\
& +\int_{\underline{t}_{1 k}}^{\bar{t}_{1 k}} e^{-m_{k}} \frac{1}{\sigma_{k}} \phi\left(\frac{z_{k}-L_{k} v_{s}}{\sigma_{k}}\right) d z_{k} \\
& +\int_{\bar{t}_{1 k}}^{\infty}\left(\Phi^{-1}\left[\frac{\Phi\left(z_{k}\right)-p_{0 k}-p_{1 k}}{\left(1-p_{0 k}-p_{1 k}\right)}\right] s_{k}+\mu_{k}\right) e^{-\left(\Phi^{-1}\left[\frac{\Phi\left(z_{k}\right)-p_{0 k}-p_{1 k}}{\left(1-p_{0 k}-p_{1 k}\right)}\right] s_{k}+\mu_{k}\right) m_{k}} \frac{1}{\sigma_{k}} \phi\left(\frac{z_{k}-L_{k} v_{s}}{\sigma_{k}}\right) d z_{k}
\end{aligned}
$$

A continuación se realizarán diversas tranformaciones a los componentes de (A.2) y (A.3) con el propósito de normalizarlos para que sean acotados. Los cinco términos de cada una de las funciones $\tau_{k}\left(m, v_{s}\right)$ y $r_{k}\left(m, v_{s}\right)$ se agrupan de acuerdo a su grado de similitud. El primer grupo corresponde al primer, tercer y quinto término; y el segundo grupo corresponde al segundo y quarto término.

$$
\begin{aligned}
& \tau_{k}\left(m, v_{s}\right)=\tau_{k}\left(m, v_{s}\right)_{G 1}+\tau_{k}\left(m, v_{s}\right)_{G 2} \\
& r_{k}\left(m, v_{s}\right)=r_{k}\left(m, v_{s}\right)_{G 1}+r_{k}\left(m, v_{s}\right)_{G 2}
\end{aligned}
$$




\section{Desarrollo algebraico del Primer Grupo}

Haciendo la transformación de variables $u_{k}=\Phi^{-1}\left[\frac{\Phi\left(z_{k}\right)}{\left(1-p_{0 k}-p_{1 k}\right)}\right] s_{k}+\mu_{k}, u_{k}=\Phi^{-1}\left[\frac{\Phi\left(z_{k}\right)-p_{0 k}}{\left(1-p_{0 k}-p_{1 k}\right)}\right] s_{k}+$ $\mu_{k}$ y $u_{k}=\Phi^{-1}\left[\frac{\Phi\left(z_{k}\right)-p_{0 k}-p_{1 k}}{\left(1-p_{0 k}-p_{1 k}\right)}\right] s_{k}+\mu_{k}$ para cada término respectivamente, resulta:

$$
\begin{aligned}
\tau_{k}\left(m, v_{s}\right)_{G 1}= & \int_{-\infty}^{0} e^{-u_{k} m_{k}} \frac{1}{\sigma_{k}} \phi\left(\frac{w_{1}\left(u_{k}\right)-L_{k} v_{s}}{\sigma_{k}}\right) \frac{\frac{\left(1-p_{0 k}-p_{1 k}\right)}{s_{k}} \phi\left(\frac{u_{k}-\mu_{k}}{s_{k}}\right)}{\phi\left(w_{1}\left(u_{k}\right)\right)} d u_{k} \\
& +\int_{0}^{1} e^{-u_{k} m_{k}} \frac{1}{\sigma_{k}} \phi\left(\frac{w_{2}\left(u_{k}\right)-L_{k} v_{s}}{\sigma_{k}}\right) \frac{\frac{\left(1-p_{0 k}-p_{1 k}\right)}{s_{k}} \phi\left(\frac{u_{k}-\mu_{k}}{s_{k}}\right)}{\phi\left(w_{2}\left(u_{k}\right)\right)} d u_{k} \\
& +\int_{1}^{\infty} e^{-u_{k} m_{k}} \frac{1}{\sigma_{k}} \phi\left(\frac{w_{3}\left(u_{k}\right)-L_{k} v_{s}}{\sigma_{k}}\right) \frac{\frac{\left(1-p_{0 k}-p_{1 k}\right)}{s_{k}} \phi\left(\frac{u_{k}-\mu_{k}}{s_{k}}\right)}{\phi\left(w_{3}\left(u_{k}\right)\right)} d u_{k} \\
r_{k}\left(m, v_{s}\right)_{G 1}= & \int_{-\infty}^{0} u_{k} e^{-u_{k} m_{k}} \frac{1}{\sigma_{k}} \phi\left(\frac{w_{1}\left(u_{k}\right)-L_{k} v_{s}}{\sigma_{k}}\right) \frac{\frac{\left(1-p_{0 k}-p_{1 k}\right)}{s_{k}} \phi\left(\frac{u_{k}-\mu_{k}}{s_{k}}\right)}{\phi\left(w_{1}\left(u_{k}\right)\right)} d u_{k} \\
& +\int_{0}^{1} u_{k} e^{-u_{k} m_{k}} \frac{1}{\sigma_{k}} \phi\left(\frac{w_{2}\left(u_{k}\right)-L_{k} v_{s}}{\sigma_{k}}\right) \frac{\frac{\left(1-p_{0 k}-p_{1 k}\right)}{s_{k}} \phi\left(\frac{u_{k}-\mu_{k}}{s_{k}}\right)}{\phi\left(w_{2}\left(u_{k}\right)\right)} d u_{k} \\
& +\int_{1}^{\infty} u_{k} e^{-u_{k} m_{k}} \frac{1}{\sigma_{k}} \phi\left(\frac{w_{3}\left(u_{k}\right)-L_{k} v_{s}}{\sigma_{k}}\right) \frac{\frac{\left(1-p_{0 k}-p_{1 k}\right)}{s_{k}} \phi\left(\frac{u_{k}-\mu_{k}}{s_{k}}\right)}{\phi\left(w_{3}\left(u_{k}\right)\right)} d u_{k}
\end{aligned}
$$

donde,

$$
\begin{aligned}
& w_{1}\left(u_{k}\right)=\Phi^{-1}\left[\Phi\left(\frac{u_{k}-\mu_{k}}{s_{k}}\right)\left(1-p_{0 k}-p_{1 k}\right)\right] \\
& w_{2}\left(u_{k}\right)=\Phi^{-1}\left[\Phi\left(\frac{u_{k}-\mu_{k}}{s_{k}}\right)\left(1-p_{0 k}-p_{1 k}\right)+p_{0 k}\right] \\
& w_{3}\left(u_{k}\right)=\Phi^{-1}\left[\Phi\left(\frac{u_{k}-\mu_{k}}{s_{k}}\right)\left(1-p_{0 k}-p_{1 k}\right)+p_{0 k}+p_{1 k}\right]
\end{aligned}
$$

Operando algebraicamente sobre el término $e^{-u_{k} m_{k}} \frac{1}{s_{k}} \phi\left(\frac{u_{k}-\mu_{k}}{s_{k}}\right)$ se obtiene:

$$
\exp \left(-\mu_{k} m_{k}+\frac{m_{k}^{2} s_{k}^{2}}{2}\right) \frac{1}{s_{k}} \phi\left(\frac{u_{k}-\left(\mu_{k}-m_{k} s_{k}^{2}\right)}{s_{k}}\right)
$$

Entonces:

$$
\begin{aligned}
\tau_{k}\left(m, v_{s}\right)_{G 1}= & n_{k} \int_{-\infty}^{0} \frac{1}{s_{k}} \phi\left(\frac{u_{k}-\left(\mu_{k}-m_{k} s_{k}^{2}\right)}{s_{k}}\right) \frac{\phi\left(\frac{w_{1}\left(u_{k}\right)-L_{k} v_{s}}{\sigma_{k}}\right)}{\phi\left(w_{1}\left(u_{k}\right)\right)} d u_{k} \\
& +n_{k} \int_{0}^{1} \frac{1}{s_{k}} \phi\left(\frac{u_{k}-\left(\mu_{k}-m_{k} s_{k}^{2}\right)}{s_{k}}\right) \frac{\phi\left(\frac{w_{2}\left(u_{k}\right)-L_{k} v_{s}}{\sigma_{k}}\right)}{\phi\left(w_{2}\left(u_{k}\right)\right)} d u_{k} \\
& +n_{k} \int_{1}^{\infty} \frac{1}{s_{k}} \phi\left(\frac{u_{k}-\left(\mu_{k}-m_{k} s_{k}^{2}\right)}{s_{k}}\right) \frac{\phi\left(\frac{w_{3}\left(u_{k}\right)-L_{k} v_{s}}{\sigma_{k}}\right)}{\phi\left(w_{3}\left(u_{k}\right)\right)} d u_{k}
\end{aligned}
$$




$$
\begin{aligned}
r_{k}\left(m, v_{s}\right)_{G 1}= & n_{k} \int_{-\infty}^{0} u_{k} \frac{1}{s_{k}} \phi\left(\frac{u_{k}-\left(\mu_{k}-m_{k} s_{k}^{2}\right)}{s_{k}}\right) \frac{\phi\left(\frac{w_{1}\left(u_{k}\right)-L_{k} v_{s}}{\sigma_{k}}\right)}{\phi\left(w_{1}\left(u_{k}\right)\right)} d u_{k} \\
& +n_{k} \int_{0}^{1} u_{k} \frac{1}{s_{k}} \phi\left(\frac{u_{k}-\left(\mu_{k}-m_{k} s_{k}^{2}\right)}{s_{k}}\right) \frac{\phi\left(\frac{w_{2}\left(u_{k}\right)-L_{k} v_{s}}{\sigma_{k}}\right)}{\phi\left(w_{2}\left(u_{k}\right)\right)} d u_{k} \\
& +n_{k} \int_{1}^{\infty} u_{k} \frac{1}{s_{k}} \phi\left(\frac{u_{k}-\left(\mu_{k}-m_{k} s_{k}^{2}\right)}{s_{k}}\right) \frac{\phi\left(\frac{w_{3}\left(u_{k}\right)-L_{k} v_{s}}{\sigma_{k}}\right)}{\phi\left(w_{3}\left(u_{k}\right)\right)} d u_{k}
\end{aligned}
$$

donde,

$$
n_{k}=\frac{\left(1-p_{0 k}-p_{1 k}\right)}{\sigma_{k}} \exp \left(-\mu_{k} m_{k}+\frac{m_{k}^{2} s_{k}^{2}}{2}\right)
$$

Haciendo la transformación de variables: $t_{k}=\frac{u_{k}-\left(\mu_{k}-m_{k} s_{k}^{2}\right)}{s_{k}}$ y dividiendo por $n_{k}$, resulta:

$$
\begin{aligned}
\tau_{k}\left(m, v_{s}\right)_{G 1}= & d_{k 1} \int_{-\infty}^{\left(m_{k} s_{k}-\mu_{k} / s\right)} \frac{\phi\left(t_{k}\right)}{d_{k 1}} \frac{\phi\left(\frac{w_{1}^{\prime}\left(t_{k}\right)-L_{k} v_{s}}{\sigma_{k}}\right)}{\phi\left(w_{1}^{\prime}\left(t_{k}\right)\right)} d t_{k} \\
& +d_{k 2} \int_{\left(m_{k} s_{k}-\mu_{k} / s\right)}^{\left(m_{k} s_{k}+\left(1-\mu_{k}\right) / s\right)} \frac{\phi\left(t_{k}\right)}{d_{k 2}} \frac{\phi\left(\frac{w_{2}^{\prime}\left(t_{k}\right)-L_{k} v_{s}}{\sigma_{k}}\right)}{\phi\left(w_{2}^{\prime}\left(t_{k}\right)\right)} d t_{k} \\
& +d_{k 3} \int_{\left(m_{k} s_{k}+\left(1-\mu_{k}\right) / s\right)}^{\infty} \frac{\phi\left(t_{k}\right)}{d_{k 3}} \frac{\phi\left(\frac{w_{3}^{\prime}\left(t_{k}\right)-L_{k} v_{s}}{\sigma_{k}}\right)}{\phi\left(w_{3}^{\prime}\left(t_{k}\right)\right)} d t_{k} \\
r_{k}\left(m, v_{s}\right)_{G 1}= & d_{k 1} \int_{-\infty}^{\left(m_{k} s_{k}-\mu_{k} / s\right)} a\left(t_{k}\right) \frac{\phi\left(t_{k}\right)}{d_{k 1}} \frac{\phi\left(\frac{\left.w_{1}^{\prime}\left(t_{k}\right)-L_{k} v_{s}\right)}{\sigma_{k}}\right)}{\phi\left(w_{1}^{\prime}\left(t_{k}\right)\right)} d t_{k} \\
& +d_{k 2} \int_{\left(m_{k} s_{k}-\mu_{k} / s\right)}^{\left(m_{k} s_{k}+\left(1-\mu_{k}\right) / s\right)} a\left(t_{k}\right) \frac{\phi\left(t_{k}\right)}{d_{k 2}} \frac{\phi\left(\frac{w_{2}^{\prime}\left(t_{k}\right)-L_{k} v_{s}}{\sigma_{k}}\right)}{\phi\left(w_{2}^{\prime}\left(t_{k}\right)\right)} d t_{k} \\
& +d_{k 3} \int_{\left(m_{k} s_{k}+\left(1-\mu_{k}\right) / s\right)}^{\infty} d t_{k} \frac{\phi\left(t_{k}\right)}{d_{k 3}} \frac{\left(\frac{w_{3}^{\prime}\left(t_{k}\right)-L_{k} v_{s}}{\sigma_{k}}\right)}{\phi\left(w_{3}^{\prime}\left(t_{k}\right)\right)}
\end{aligned}
$$

donde,

$$
\begin{aligned}
& d_{k 1}=\Phi\left(-\frac{\mu_{k}}{s_{k}}-m_{k} s_{k}\right)-\Phi(-\infty) \\
& d_{k 2}=\Phi\left(\frac{1-\mu_{k}}{s_{k}}-m_{k} s_{k}\right)-\Phi\left(-\frac{\mu_{k}}{s_{k}}-m_{k} s_{k}\right) \\
& d_{k 3}=\Phi(\infty)-\Phi\left(\frac{1-\mu_{k}}{s_{k}}-m_{k} s_{k}\right)
\end{aligned}
$$




$$
\begin{array}{cc}
w_{1}^{\prime}\left(t_{k}\right)= & \Phi^{-1}\left[\Phi\left(t_{k}-m_{k} s_{k}\right)\left(1-p_{0 k}-p_{1 k}\right)\right] \\
w_{2}^{\prime}\left(t_{k}\right)= & \Phi^{-1}\left[\Phi\left(t_{k}-m_{k} s_{k}\right)\left(1-p_{0 k}-p_{1 k}\right)+p_{0 k}\right] \\
w_{3}^{\prime}\left(t_{k}\right)= & \Phi^{-1}\left[\Phi\left(t_{k}-m_{k} s_{k}\right)\left(1-p_{0 k}-p_{1 k}\right)+p_{0 k}+p_{1 k}\right] \\
a\left(t_{k}\right)=\left(t_{k} s_{k}+\mu_{k}-m_{k} s_{k}^{2}\right)
\end{array}
$$

Multiplicando y dividiendo por $\phi\left(\frac{t_{k}-m_{k} s_{k}-L_{k} v_{s}}{\sigma_{k}}\right) \phi\left(\frac{t_{k}-m_{k} s_{k}}{\sigma_{k}}\right)$, resulta:

$$
\begin{aligned}
& \tau_{k}\left(m, v_{s}\right)_{G 1}= \\
& \exp \left(-m_{k} s_{k}\left(m_{k} s_{k}\left(1-\sigma_{k}^{2}\right)+2 L_{k} v_{s}\right)\right) d_{k 1} n_{k} \int_{-\infty}^{\left(m_{k} s_{k}-\mu_{k} s_{k}\right)} \frac{1}{d_{k 1}} \phi\left(\frac{t_{k}-m_{k} s_{k}\left(1-\sigma_{k}^{2}\right)-L_{k} v_{s}}{\sigma_{k}}\right) \\
& \frac{\phi\left(t_{k}-m_{k} s_{k}\right)}{\phi\left(\frac{t_{k}-m_{k} s_{k}-L_{k} v_{s}}{\sigma_{k}}\right)} \frac{\phi\left(\frac{w_{1}^{\prime}\left(t_{k}\right)-L_{k} v_{s}}{\sigma_{k}}\right)}{\phi\left(w_{1}^{\prime}\left(t_{k}\right)\right)} d t_{k} \\
& +\exp \left(-m_{k} s_{k}\left(m_{k} s_{k}\left(1-\sigma_{k}^{2}\right)+2 L_{k} v_{s}\right)\right) d_{k 2} n_{k} \int_{\left(m_{k} s_{k}-\mu_{k} / s\right)}^{\left(m_{k} s_{k}+\left(1-\mu_{k}\right) / s\right)} \frac{1}{d_{k 2}} \phi\left(\frac{t_{k}-m_{k} s_{k}\left(1-\sigma_{k}^{2}\right)-L_{k} v_{s}}{\sigma_{k}}\right) \\
& \frac{\phi\left(t_{k}-m_{k} s_{k}\right)}{\phi\left(\frac{t_{k}-m_{k} s_{k}-L_{k} v_{s}}{\sigma_{k}}\right)} \frac{\phi\left(\frac{w_{2}^{\prime}\left(t_{k}\right)-L_{k} v_{s}}{\sigma_{k}}\right)}{\phi\left(w_{2}^{\prime}\left(t_{k}\right)\right)} d t_{k} \\
& +\exp \left(-m_{k} s_{k}\left(m_{k} s_{k}\left(1-\sigma_{k}^{2}\right)+2 L_{k} v_{s}\right)\right) d_{k 3} n_{k} \int_{\left(m_{k} s_{k}+\left(1-\mu_{k}\right) / s\right)}^{\infty} \frac{1}{d_{k 3}} \phi\left(\frac{t_{k}-m_{k} s_{k}\left(1-\sigma_{k}^{2}\right)-L_{k} v_{s}}{\sigma_{k}}\right) \\
& \frac{\phi\left(t_{k}-m_{k} s_{k}\right)}{\phi\left(\frac{t_{k}-m_{k} s_{k}-L_{k} v_{s}}{\sigma_{k}}\right)} \frac{\phi\left(\frac{w_{3}^{\prime}\left(t_{k}\right)-L_{k} v_{s}}{\sigma_{k}}\right)}{\phi\left(w_{3}^{\prime}\left(t_{k}\right)\right)} d t_{k}
\end{aligned}
$$




$$
\begin{aligned}
& r_{k}\left(m, v_{s}\right)_{G 1}= \\
& \exp \left(-m_{k} s_{k}\left(m_{k} s_{k}\left(1-\sigma_{k}^{2}\right)+2 L_{k} v_{s}\right)\right) d_{k 1} n_{k} \int_{-\infty}^{\left(m_{k} s_{k}-\mu_{k} / s_{k}\right)} a\left(t_{k}\right) \frac{1}{d_{k 1}} \phi\left(\frac{t_{k}-m_{k} s_{k}\left(1-\sigma_{k}^{2}\right)-L_{k} v_{s}}{\sigma_{k}}\right) \\
& \frac{\phi\left(t_{k}-m_{k} s_{k}\right)}{\phi\left(\frac{t_{k}-m_{k} s_{k}-L_{k} v_{s}}{\sigma_{k}}\right)} \frac{\phi\left(\frac{w_{1}^{\prime}\left(t_{k}\right)-L_{k} v_{s}}{\sigma_{k}}\right)}{\phi\left(w_{1}^{\prime}\left(t_{k}\right)\right)} d t_{k} \\
& +\exp \left(-m_{k} s_{k}\left(m_{k} s_{k}\left(1-\sigma_{k}^{2}\right)+2 L_{k} v_{s}\right)\right) d_{k 2} n_{k} \int_{\left(m_{k} s_{k}-\mu_{k} / s\right)}^{\left(m_{k} s_{k}+\left(1-\mu_{k}\right) / s\right)} a\left(t_{k}\right) \frac{1}{d_{k 2}} \phi\left(\frac{t_{k}-m_{k} s_{k}\left(1-\sigma_{k}^{2}\right)-L_{k} v_{s}}{\sigma_{k}}\right) \\
& \frac{\phi\left(t_{k}-m_{k} s_{k}\right)}{\phi\left(\frac{t_{k}-m_{k} s_{k}-L_{k} v_{s}}{\sigma_{k}}\right)} \frac{\phi\left(\frac{w_{2}^{\prime}\left(t_{k}\right)-L_{k} v_{s}}{\sigma_{k}}\right)}{\phi\left(w_{2}^{\prime}\left(t_{k}\right)\right)} d t_{k} \\
& +\exp \left(-m_{k} s_{k}\left(m_{k} s_{k}\left(1-\sigma_{k}^{2}\right)+2 L_{k} v_{s}\right)\right) d_{k 3} n_{k} \int_{\left(m_{k} s_{k}+\left(1-\mu_{k}\right) / s\right)}^{\infty} a\left(t_{k}\right) \frac{1}{d_{k 3}} \phi\left(\frac{t_{k}-m_{k} s_{k}\left(1-\sigma_{k}^{2}\right)-L_{k} v_{s}}{\sigma_{k}}\right) \\
& \frac{\phi\left(t_{k}-m_{k} s_{k}\right)}{\phi\left(\frac{t_{k}-m_{k} s_{k}-L_{k} v_{s}}{\sigma_{k}}\right)} \frac{\phi\left(\frac{w_{3}^{\prime}\left(t_{k}\right)-L_{k} v_{s}}{\sigma_{k}}\right)}{\phi\left(w_{3}^{\prime}\left(t_{k}\right)\right)} d t_{k}
\end{aligned}
$$

Haciendo la transformación de variables: $t^{*}=\frac{t_{k}-m_{k} s_{k}\left(1-\sigma_{k}\right)-L_{k} v_{s}}{\sigma_{k}} ; t_{k}=t^{*} \sigma_{k}+m_{k} s_{k}\left(1-\sigma_{k}\right)+L_{k} v_{s}$, resulta:

$$
\begin{aligned}
& \tau_{k}\left(m, v_{s}\right)_{G 1}= \\
& b d_{k 1} \int_{-\infty}^{m_{k} s_{k}-\frac{\mu_{k} / s+L_{k} v_{s}}{\sigma_{k}}} \frac{\phi\left(t_{k}^{*}\right)}{d_{k 1}} \frac{\phi\left(\left(t_{k}^{*}-m_{k} s_{k}\right) \sigma_{k}+L_{k} v_{s}\right)}{\phi\left(t^{*}-m_{k} s_{k}\right)} \frac{\phi\left(\frac{w_{1}^{\prime \prime}\left(t_{k}^{*}\right)-L_{k} v_{s}}{\sigma_{k}}\right)}{\phi\left(w_{1}^{\prime \prime}\left(t_{k}^{*}\right)\right)} d t_{k}^{*} \\
& +b d_{k 2} \int_{m_{k} s_{k}-\frac{\mu_{k} / s+L_{k} v_{s}}{\sigma_{k}}}^{m_{k} s_{k}+\frac{\left(1-\mu_{k}\right) / s+L_{k} v_{s}}{\sigma_{k}}} \frac{\phi\left(t_{k}^{*}\right)}{d_{k 2}} \frac{\phi\left(\left(t_{k}^{*}-m_{k} s_{k}\right) \sigma_{k}+L_{k} v_{s}\right)}{\phi\left(t^{*}-m_{k} s_{k}\right)} \frac{\phi\left(\frac{w_{2}^{\prime \prime}\left(t_{k}^{*}\right)-L_{k} v_{s}}{\sigma_{k}}\right)}{\phi\left(w_{2}^{\prime \prime}\left(t_{k}^{*}\right)\right)} d t_{k}^{*} \\
& +b d_{k 3} \int_{m_{k} s_{k}+\frac{\left(1-\mu_{k}\right) / s+L_{k} v_{s}}{\sigma_{k}}}^{\infty} \frac{\phi\left(t_{k}^{*}\right)}{d_{k 3}} \frac{\phi\left(\left(t_{k}^{*}-m_{k} s_{k}\right) \sigma_{k}+L_{k} v_{s}\right)}{\phi\left(t^{*}-m_{k} s_{k}\right)} \frac{\phi\left(\frac{w_{3}^{\prime \prime}\left(t_{k}^{*}\right)-L_{k} v_{s}}{\sigma_{k}}\right)}{\phi\left(w_{3}^{\prime \prime}\left(t_{k}^{*}\right)\right)} d t_{k}^{*}
\end{aligned}
$$




$$
\begin{aligned}
& r_{k}\left(m, v_{s}\right)_{G 1}= \\
& b d_{k 1} \int_{-\infty}^{m_{k} s_{k}-\frac{\mu_{k} / s+L_{k} v_{s}}{\sigma_{k}}} a^{\prime}\left(t_{k}^{*}\right) \frac{\phi\left(t_{k}^{*}\right)}{d_{k 1}} \frac{\phi\left(\left(t_{k}^{*}-m_{k} s_{k}\right) \sigma_{k}+L_{k} v_{s}\right)}{\phi\left(t^{*}-m_{k} s_{k}\right)} \frac{\phi\left(\frac{w_{1}^{\prime \prime}\left(t_{k}^{*}\right)-L_{k} v_{s}}{\sigma_{k}}\right)}{\phi\left(w_{1}^{\prime \prime}\left(t_{k}^{*}\right)\right)} d t_{k}^{*} \\
& +b d_{k 2} \int_{m_{k} s_{k}-\frac{\mu_{k} / s+L_{k} v_{s}}{\sigma_{k}}}^{m_{k} s_{k}+\frac{\left(1-\mu_{k}\right) / s+L_{k} v_{s}}{\sigma_{k}}} a^{\prime}\left(t_{k}^{*}\right) \frac{\phi\left(t_{k}^{*}\right)}{d_{k 2}} \frac{\phi\left(\left(t_{k}^{*}-m_{k} s_{k}\right) \sigma_{k}+L_{k} v_{s}\right)}{\phi\left(t^{*}-m_{k} s_{k}\right)} \frac{\phi\left(\frac{w_{2}^{\prime \prime}\left(t_{k}^{*}\right)-L_{k} v_{s}}{\sigma_{k}}\right)}{\phi\left(w_{2}^{\prime \prime}\left(t_{k}^{*}\right)\right)} d t_{k}^{*} \\
& +b d_{k 3} \int_{m_{k} s_{k}+\frac{\left(1-\mu_{k}\right) / s+L_{k} v_{s}}{\sigma_{k}}}^{\infty} a^{\prime}\left(t_{k}^{*}\right) \frac{\phi\left(t_{k}^{*}\right)}{d_{k 3}} \frac{\phi\left(\left(t_{k}^{*}-m_{k} s_{k}\right) \sigma_{k}+L_{k} v_{s}\right)}{\phi\left(t^{*}-m_{k} s_{k}\right)} \frac{\phi\left(\frac{w_{3}^{\prime \prime}\left(t_{k}^{*}\right)-L_{k} v_{s}}{\sigma_{k}}\right)}{\phi\left(w_{3}^{\prime \prime}\left(t_{k}^{*}\right)\right)} d t_{k}^{*}
\end{aligned}
$$

donde,

$$
\begin{gathered}
b=\exp \left(-m_{k} s_{k}\left(m_{k} s_{k}\left(1-\sigma_{k}^{2}\right)+2 L_{k} v_{s}\right)\right) \sigma_{k} \\
a^{\prime}\left(t_{k}^{*}\right)=\left[\left(t_{k}^{*}-m_{k} s_{k}\right) \sigma_{k}+L_{k} v_{s}\right] s_{k}+\mu_{k} \\
w_{1}^{\prime \prime}\left(t_{k}^{*}\right)=\Phi^{-1}\left[\Phi\left(\left(t_{k}^{*}-m_{k} s_{k}\right) \sigma_{k}+L_{k} v_{s}\right)\left(1-p_{0 k}-p_{1 k}\right)\right] \\
w_{2}^{\prime \prime}\left(t_{k}^{*}\right)=\Phi^{-1}\left[\Phi\left(\left(t_{k}^{*}-m_{k} s_{k}\right) \sigma_{k}+L_{k} v_{s}\right)\left(1-p_{0 k}-p_{1 k}\right)+p_{0 k}\right] \\
w_{3}^{\prime \prime}\left(t_{k}^{*}\right)=\Phi^{-1}\left[\Phi\left(\left(t_{k}^{*}-m_{k} s_{k}\right) \sigma_{k}+L_{k} v_{s}\right)\left(1-p_{0 k}-p_{1 k}\right)+p_{0 k}+p_{1 k}\right]
\end{gathered}
$$

\section{Desarrollo algebraico del Segundo Grupo}

Haciendo la transformación de variables $u_{k}=z_{k} \mathrm{y}$ dividiendo por $n_{k}$, resulta:

$$
\begin{aligned}
\tau_{k}\left(m, v_{s}\right)_{G 2}= & n_{k}^{-1} \int_{\underline{t}_{0 k}}^{\bar{t}_{0 k}} \frac{1}{\sigma_{k}} \phi\left(\frac{u_{k}-L_{k} v_{s}}{\sigma_{k}}\right) d u_{k} \\
& +n_{k}^{-1} e^{-m_{k}} \int_{\underline{t}_{1 k}}^{\bar{t}_{1 k}} \frac{1}{\sigma_{k}} \phi\left(\frac{u_{k}-L_{k} v_{s}}{\sigma_{k}}\right) d u_{k} \\
r_{k}\left(m, v_{s}\right)_{G 2}= & n_{k}^{-1} \int_{\underline{t}_{0 k}}^{\bar{t}_{0 k}} 0 \frac{1}{\sigma_{k}} \phi\left(\frac{u_{k}-L_{k} v_{s}}{\sigma_{k}}\right) d u_{k} \\
& +n_{k}^{-1} e^{-m_{k}} \int_{\underline{t}_{1 k}}^{\bar{t}_{1 k}} \frac{1}{\sigma_{k}} \phi\left(\frac{u_{k}-L_{k} v_{s}}{\sigma_{k}}\right) d u_{k}
\end{aligned}
$$

